\title{
Numerical analysis of thermoacoustically driven thermoacoustic refrigerator with a stack of parallel plates having corrugated surfaces
}

Zahra Bouramdane ${ }^{1 *}$, Abdellah Bah ${ }^{1}$, Mohammed Alaoui ${ }^{1}$ and Nadia Martaj ${ }^{2}$

\begin{abstract}
Most numerical research on thermoacoustic devices with a stack made of parallel plates has considered a rectangular form for the plate. However, a variety of plate shapes can improve the heat transfer and performance of the stack. In this paper, a 2D numerical model based on computational fluid dynamics (CFD) analysis is used to examine the efficiency of thermoacoustic couples using a diversity of plate surfaces. For this investigation, flat plates and others profiles with corrugated surfaces (rounded and triangular surfaces) were tested to compare their performances and the effect of plate form on thermoacoustic systems. The efficiency of the thermoacoustic engine (TAE) is measured in terms of the generated acoustic pressure and the performance of the thermoacoustic refrigerator (TAR) is considered in terms of the temperature gradient along the refrigeration stack. The results showed that plates profile with irregular surfaces improves the generated acoustic pressure due to the increase of the stack porosity known as the blockage ratio (BR). The triangular shape performed better with a 10\% gain than using a flat plate, followed by rounded ripples with a $5.2 \%$ increase in pressure amplitude compared to flat surfaces. Unlike in TAR, it was revealed that the stack made of flatform could produce a higher temperature difference $(\Delta T)$ among the refrigeration stack extremities. By using rounded ripples, $\Delta T$ undergoes a reduction up to $52 \%$, and $65 \%$ if using triangular ripples compared to flatform. From this investigation, it is observed that the stack with corrugated plates is the most suitable for TAE; however, the flat plates generate a higher temperature difference in TAR.
\end{abstract}

Keywords: Thermoacoustic, Refrigerator, Engine, Plates form, CFD

0.0.0.1 Greek symbols $v$ Kinematic viscosity, $\mathrm{m}^{2} . \mathrm{s}^{-1}$

$\gamma$ Ratio of specific heat

$\alpha$ Thermal diffusivity, $\mathrm{m}^{2} . \mathrm{s}^{-1}$

$\beta$ Thermal expansion coefficient, $\mathrm{K}^{-1}$

$\delta_{\mathrm{v}}$ Viscous penetration depth, $\mathrm{m}$

$\delta_{\mathrm{k}}$ Thermal penetration depth, $\mathrm{m}$

$\omega$ Angular frequency, rad. $\mathrm{s}^{-1}$

$\Gamma$ Normalized temperature gradient

* Correspondence: zahra.bouramdane@gmail.com

${ }^{1}$ Ecole Nationale Superieure des Arts et Metiers Rabat (ENSAM), Mohammed V University of Rabat, Avenue de l'Armee Royale, Madinat Al Irfane 10100,

B.P. 6207 Rabat-Instituts, Rabat, Morocco

Full list of author information is available at the end of the article $\varepsilon_{\mathrm{s}}$ Plate heat capacity ratio

$\rho$ Density, $\mathrm{kg} \mathrm{m}^{-3}$

$\mu$ Dynamic viscosity, kg. $\mathrm{m}^{-1} \cdot \mathrm{s}^{-1}$

0.0.0.2 Subscripts, superscripts v Viscous

Se Stack engine

Sr Stack refrigerator

s Stack

$r$ Resonator

n Normalized

m Mean

h Hot 
c Cold

crit Critical

amb Ambient

\section{Introduction}

The stack is the heart of thermoacoustic machines: it is a solid, rigid, and porous structure, which supports high temperature gradients. The first stacks used consisted of a sheet of plastic material coiled in a spiral with fishing line spacers between each layer [1], some stacks consist of parallel plates, for example, stainless steel plates [2], honeycomb-shaped stacks, also made of stainless steel [3], and ceramic monoliths with a regular structure of parallel square channels are also used [4]. Research has been carried out on stacks in the form of pin-array stacks $[5,6]$. These studies show that this type of stack significantly increases the efficiency of thermoacoustic machines compared to other types of stacks. Bôsel et al. suggest using a stack of plates made up of short sections oriented randomly with respect to each other as a new attempt for achieving higher power densities [7]. This alternative stack arrangement was expected to benefit from improved heat transfer characteristics due to the absence of axial conduction within the stack; axial conduction limits the temperature gradient achievable. It is found that the power density was raised by approximately $50 \%$. The coefficient of performance defined as useful heat output divided by work input increased by approximately one third. Stacks made from slices of porous material were also tested by the use of glassy carbon foam [8, 9]. Zoontjens et al. [10] conducted a numerical investigation on the performance of thermoacoustic devices with a diversity of plate edge shapes. The results show that plate shapes with blunter and enlarged profiles retard the rate of heat conduction at higher drive ratios but at low drive ratios, the rate of heat transfer increases. Matveev [11] established a basic approach based on Rayleigh's criterion for estimating energy conversion in thermoacoustic transverse-pin and tortuous stacks. The results indicate that this approach offers fast approximations for efficiency and energy conversion in thermoacoustic systems. However, it is limited by the necessity of having a great acoustic displacement comparative to the gap among pins. Asgharian and Matveev [12] modeled moving gas parcels in a transverse-pin array stack with a Lagrangian approach. The results indicate that the stack with transverse-pin improves the performance of thermoacoustic energy conversion compared to devices with other stacks. Zolpakar and Mohd-Ghazali [13, 14] conducted a simultaneous optimization of four different geometrical variables and three different materials (hand fabricated Mylar stack, off-the-shelf Celcor substrates, and a 3D printed stack) of a thermoacoustic refrigerator stack using experiments and the MOGA (multi-objective genetic algorithm). The results displayed that, with a resonator diameter of $D=$ $3.8 \mathrm{~cm}$, acoustic power of $\mathrm{Wn}=4.86 \mathrm{~W}$, and cooling power of $Q_{c}=6.57 \mathrm{~W}$, the optimum coefficient of performance was $\mathrm{COP}=1.35$. It is also indicated that the best performance was achieved with the $3 \mathrm{D}$ printed stack, with a temperature at the cold end of Tc $=18.9^{\circ} \mathrm{C}$ and a temperature difference across the stack of $\Delta \mathrm{T}=$ $18.1^{\circ} \mathrm{C}$. Dragonetti et al. [15] investigated the efficiency of a TAE in which the stack is modeled as a tortuous porous material. The reported results showed that pore tortuosity disturbs the acoustic energy and the viscous losses generated in the stack with respect to thermal losses. Abd El-Rahman et al. [16] conducted a CFD investigation on a half-wavelength TAR with a stack formed of rectangular pores. The simulation provides a developed 3D finitevolume approach to study the heat exchange between the working fluid and the adjacent surfaces in the stack of rectangular pores. Yahya et al. [17] carried out an experimental study on a thermoacoustic refrigerator to determine the thermal performance of random stack materials. The results show that the steel wool stacks, among the tested random stack materials, reached the optimal cooling power and the lowest temperature. It is also shown that the stack made of Mylar sheets revealed a higher efficiency in comparison to both stacks made of random material and parallel plate stack. It attained a temperature difference and maximums COP of $7.7^{\circ} \mathrm{C}$ and 0.217 , respectively. Napolitano et al. [18] studied the efficiency of a stack made of open-cell foam to increase the energy conversion inside a standing wave thermoacoustic engine. The outcomes were compared with those found by regular stack made with straight holes whose cross-sections have regular forms. The investigation exposes that, for the considered operating conditions, the stack made with partially reticulated open-cell foams has shown a high performance compared with the commonly used stacks and the fully reticulated open-cell foams. Liu et al. [19] conducted a study on a standing wave TAE with a stack made of converging plates. The results display that picking an appropriate value of the ratio of the plate gap to the thermal penetration depth at the cold extremity of the stack in the parallel and the converging stacks can enhance the acoustic energy and the system performance. It is also shown that with the same ratio, the parallel plates stack can be replaced by the converging model with a decrease in the cost of consumptive materials. Chaiwongsa and Wongwises [20] analyzed the influence of a circular stack with different blockage ratios on the performance of a standing wave TAR using heat pipes. It is found that the optimum stack geometry for standing wave TARs is the circular stack with a blockage ratio of 0.71 ; it generates the highest temperature difference and the lowest cold temperature.

The performance of thermoacoustic refrigerator systems has been continuously improved. As presented 
above, various studies have been carried out to investigate the role of the stack and the effect of its pore shape on the performance of thermoacoustic engines and refrigerators by testing many geometries that the stack can have; the most regularly used are the parallel plates, spiral, corning celcor, and honeycomb, as exposed in Fig. 1. The analysis of the above-cited literature shows that no work concerning the analysis on the influence of plate surface shape on the performance of thermoacoustic systems has been reported except Zoontjens et al. [10] who was limited to studying plate edge profiles, as discussed above. In this paper, a two-dimensional (2D) model, based on CFD simulation carried out using the software COMSOL Multiphysics, is performed to study and examine the performance of a standing wave TAE and a thermally driven TAR comprising a stack of parallel pales. The use of CFD as an analysis tool in thermoacoustics is largely underused, and this tool allows a detailed study of the thermoacoustic effect which is difficult to achieve through experimentation. The novelty of the work is the use of a parallel plate stack made of plates with a corrugated surface. Three shapes of plate surface were tested, flat plates (considered a better reference, especially for comparison with plates of different shapes), plates with rounded ripples (the surface of the plates is formed by a series of circular corrugations of the same diameter), and plates with triangular ripples (the surface of the plates is formed by a series of triangular corrugations). The choice of these shapes is based on the increase in the flow impedance around the plates, which could improve the performance of the stack by constraining the gas packages at the level of the plates. The results from the comparison of many stack geometries may guide the development of this kind of system. Therefore, the purpose of this study is to introduce a thermally driven TAR and to show that a constant temperature gradient is sufficient to sustain strong oscillations that lead to heat pumping between the ends of the refrigerator stack. The effects of stack geometry and blockage ratio on engine efficiency and refrigerator performance are given. The efficiency of TAE is measured in terms of the generated acoustic pressure and the performance of TAR is measured in terms of the temperature difference across the refrigeration stack and the temperature at the cold side of the refrigeration stack.

\section{Stack of parallel plates}

In this part, the analysis of a stack formed with parallel plates where the solid plates have a spacing of $2 y_{0}$ and a thickness of $2 l_{0}$ is presented. The fluid is assumed to have an arbitrary viscosity with the Prandtl number Pr and the stack is oriented in the direction of the acoustic oscillations $(x)$ as shown in Fig. 2. We denote $c_{p}, c_{s}, k$, and $\mathrm{k}_{\mathrm{s}}$ the heat capacity of the fluid at constant pressure, the heat capacity of the stack material, the fluid, and solid plate's thermal conductivities respectively. The equations governing the analysis are:

- Continuity equation:

$$
\frac{\partial \rho}{\partial t}+\nabla \cdot(\rho v)=0
$$

- Momentum conservation:

$\rho\left(\frac{\partial \mathrm{v}}{\partial \mathrm{t}}+(\mathrm{v} \cdot \nabla) \mathrm{v}\right)=-\nabla_{\mathrm{P}}+\mu \nabla^{2} \mathrm{v}$

- The energy equation for the fluid:

$$
\rho \mathrm{T}\left(\frac{\partial \mathrm{s}}{\partial \mathrm{t}}+\mathrm{v} \cdot \nabla \mathrm{s}\right)=\nabla \cdot(\mathrm{k} \nabla \mathrm{T})
$$

\section{- The energy equation for the plate:}
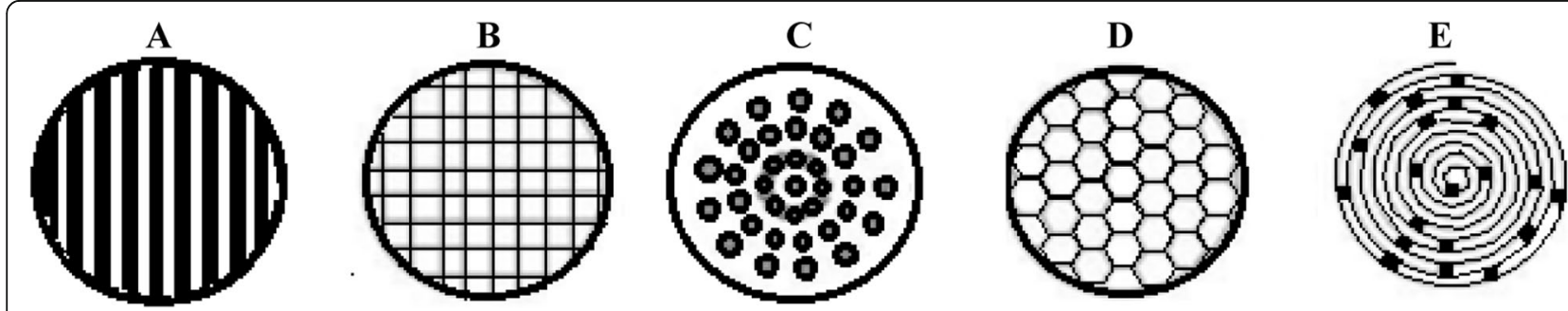

Fig. 1 Stack geometries A parallel plate, B corning celcor, C pin array, D.honeycomb, and $\mathbf{E}$ spiral stack [21] 

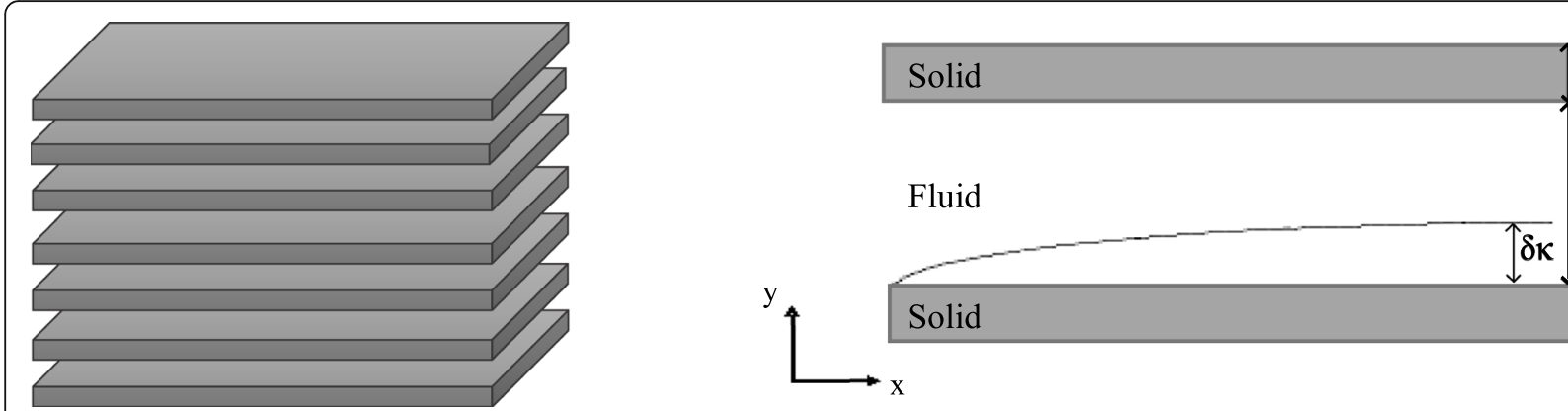

Fig. 2 Stack of parallel plates. The thermal penetration depth $\delta k$, into the surrounding fluid, is symbolically modeled

$$
\rho_{\mathrm{s}} \mathrm{c}_{\mathrm{s}} \frac{\partial \mathrm{T}_{\mathrm{s}}}{\partial \mathrm{t}}=\mathrm{K}_{\mathrm{s}} \nabla^{2} \mathrm{~T}_{\mathrm{s}}
$$

The acoustic and thermodynamic variables used for the description of the behavior of the system are the density $\rho$, the temperature $T$, the pressure $P$, the particle velocity $\mathrm{u}$, the mass entropy $\mathrm{s}$, and the temperature of the solid $\mathrm{T}_{\mathrm{S}}$. In the context of a linear description, these variables are written in the following form, the time dependence is usually chosen in $\mathrm{e}^{\mathrm{i} \omega \mathrm{t}}$ :

$$
\begin{aligned}
& \mathrm{p}=\mathrm{p}_{\mathrm{m}}+\mathrm{p}_{1}(\mathrm{x}) \mathrm{e}^{\mathrm{i} \omega \mathrm{t}} \\
& \rho=\rho_{\mathrm{m}}(\mathrm{x})+\rho_{1}(\mathrm{x}, \mathrm{y}) \mathrm{e}^{\mathrm{i} \omega \mathrm{t}} \\
& \mathrm{u}=\hat{\mathrm{x}} \mathrm{u}_{1}(\mathrm{x}, \mathrm{y}) \mathrm{e}^{\mathrm{i} \omega \mathrm{t}}+\hat{\mathrm{y}} \mathrm{v}_{1}(\mathrm{x}, \mathrm{y}) \mathrm{e}^{\mathrm{i} \omega \mathrm{t}} \\
& \mathrm{T}=\mathrm{T}_{\mathrm{m}}(\mathrm{x})+\mathrm{T}_{1}(\mathrm{x}, \mathrm{y}) \mathrm{e}^{\mathrm{i} \omega \mathrm{t}} \\
& \mathrm{T}_{\mathrm{s}}=\mathrm{T}_{\mathrm{m}}(\mathrm{x})+\mathrm{T}_{\mathrm{s} 1}(\mathrm{x}, \mathrm{y}) \mathrm{e}^{\mathrm{i} \omega \mathrm{t}} \\
& \mathrm{s}=\mathrm{s}_{\mathrm{m}}(\mathrm{x})+\mathrm{s}_{1}(\mathrm{x}, \mathrm{y}) \mathrm{e}^{\mathrm{i} \omega \mathrm{t}}
\end{aligned}
$$

Replacing these terms in the main equations of fluid motion and by integrating the resulting expressions in the region of the stack, we obtain the thermoacoustic wave equation and the equation of energy flow through the cross section of the stack:

$$
\begin{aligned}
1+\left(\frac{(\gamma-1) f_{k}}{1+\varepsilon_{s}}\right) p_{1}+\frac{\rho_{m} a^{2}}{\omega^{2}} \frac{d}{d x}\left(\frac{1-f_{v}}{\rho_{m}} \frac{d p_{1}}{d x}\right)-\beta \frac{a^{2}}{\omega^{2}} \frac{f_{k}-f_{v}}{(1-\mathrm{P} r)\left(1+\varepsilon_{s}\right)} \frac{d T_{m}}{d x} \frac{d p_{1}}{d x}=0 \\
H_{2}=\frac{\Pi y_{0}}{2 \omega \rho_{m}} \operatorname{Im}\left[\frac{d \tilde{p}_{1}}{d x} p_{1}\left(1-\tilde{f}_{v}-\frac{T_{m} \beta\left(f_{k}-\tilde{f}_{v}\right)}{(1+\operatorname{Pr})\left(1+\varepsilon_{s}\right)}\right)\right] \\
\quad+\frac{\Pi y_{0} c_{p}}{2 \omega^{3} \rho_{m}(1-\operatorname{Pr})} \frac{d T_{m}}{d x} \frac{d p_{1}}{d x} \frac{d \tilde{p}_{1}}{d x} \\
\quad \times \operatorname{Im}\left[\tilde{f}_{v}+\frac{\left(f_{k}-\tilde{f}_{v}\right)\left(1+\varepsilon_{s} f_{v} / f_{k}\right)}{(1+\operatorname{Pr})\left(1+\varepsilon_{s}\right)}\right]-\Pi\left(y_{0} k+l k_{s}\right) \frac{d T_{m}}{d x}
\end{aligned}
$$

Swift [22] describes the thermoacoustic effect and presents the normalized equations of the acoustic power generated in TAE $\left(W_{n}\right)$ and the overall cooling power obtained in TAR $\left(\mathrm{Q}_{\mathrm{cn}}\right)$ as follows:

$$
\begin{aligned}
W_{n} & \approx \frac{1}{4 \gamma} \delta_{k n} D R^{2} L_{s n}\left[B R(\gamma-1) \cos ^{2}\left(x_{c n}\right)\left(\frac{\Gamma}{(1+\sqrt{\operatorname{Pr}}) \Lambda}-1\right)-\frac{\sin ^{2}\left(x_{c n}\right) \sqrt{\operatorname{Pr}}}{B R \times \Lambda}\right] \\
\mathrm{Q}_{\mathrm{cn}} & \approx-\left\{\frac{\delta_{\mathrm{kn}} D R^{2} \sin \left(2 \mathrm{x}_{\mathrm{sn}}\right)}{8 \gamma(1+\operatorname{Pr})\left(1-\sqrt{\operatorname{Pr}} \delta_{\mathrm{kn}}+\frac{1}{2} \operatorname{Pr} \delta_{\mathrm{kn}}^{2}\right)}\right\} \\
& \times\left[\frac{\Delta \mathrm{T}_{\mathrm{mn}} \tan \left(\mathrm{x}_{\mathrm{sn}}\right)}{(\gamma-1) \mathrm{BRL} L_{\mathrm{sn}}} \times \frac{1+\operatorname{Pr}+\sqrt{\operatorname{Pr}}}{1+\sqrt{\operatorname{Pr}}}-\left(1+\sqrt{\operatorname{Pr}}-\sqrt{\operatorname{Pr}} \delta_{\mathrm{kn}}\right)\right]
\end{aligned}
$$

where $\mathrm{f}_{\mathrm{k}}, \mathrm{f}_{\mathrm{v}}, \varepsilon_{\mathrm{s}}, \Gamma$, and BR are the thermoviscous function called the Rott's functions for temperature and viscosity, the heat capacity correction factor of the plates, the normalized temperature gradient, and the porosity (blockage ratio) known as the working fluid area to the total stack area given by:

$$
\begin{aligned}
& f_{k}=\frac{\tanh \left[(1+\mathrm{i}) \mathrm{y}_{0} / \delta_{\mathrm{k}}\right]}{(1+i) y_{0} / \delta_{k}} \\
& f_{v}=\frac{\tanh \left[(1+\mathrm{i}) \mathrm{y}_{0} / \delta_{v}\right]}{(1+i) y_{0} / \delta_{v}} \\
& \Gamma=\frac{\nabla T_{n}}{B R(\gamma-1) L_{s n}} \tan \left(x_{n}\right) \\
& \varepsilon_{s}=\sqrt{\frac{\rho_{m} c_{p} K}{\rho_{\text {solid }} c_{\text {solid }} K_{\text {solid }}}} \\
& B R=\frac{A_{g}}{A_{s}}=\frac{y_{0}}{y_{0+l_{0}}}
\end{aligned}
$$

and $\Lambda$ is defined as

$$
\Lambda=1-\frac{\delta_{v}}{y_{0}}+\frac{\delta_{v}{ }^{2}}{2 y_{0}^{2}}
$$

The gap between the working fluid particles and the plate solid surfaces is an important factor to be 
considered concerning the approach of heat transfer process. For the fluid particle in direct interaction with the solid surfaces $\left(\mathrm{y}<<\delta_{\mathrm{K}}\right)$, the heat transfer is of an isothermal nature. However, distant from the solid walls $\left(\mathrm{y}>>\delta_{\mathrm{k}}\right)$, the process is of adiabatic nature. In fact, only the particles placed at a distance equal to a thermal boundary layer thickness $\left(\delta_{\mathrm{K}}\right)$ ensure the process of thermoacoustic energy conversion. The heat transfer is provided by $\delta_{\mathrm{k}}$. However, it is discouraged by the viscous boundary layer $\left(\delta_{v}\right)$ that arises next to the plate where losses due to viscous effect occur. $\delta_{\mathrm{K}}$ and $\delta_{\mathrm{v}}$ are defined as:

$$
\begin{aligned}
& \delta_{k}=\sqrt{\frac{2 K}{\rho C_{p \omega}}} \\
& \delta_{v}=\sqrt{\frac{2 \mu}{\rho \omega}}
\end{aligned}
$$

The form of the stack is described in Rott's function $\mathrm{f}_{\mathrm{k}}$ as a function of the ratio between hydraulic radiuses, $r$ (expressed as the ratio of the cross-sectional area and the perimeter of the channel) and thermal penetration depth $\delta_{\mathrm{k}}$ of different stack geometry. As shown to Fig. 3, pin array stack geometry performed better; however, such a stack poses a technical problem of realization knowing that it is necessary to build a three-dimensional structure of regularly spaced parallel rods whose diameter is submillimeter. The next best performing profile is parallel plates.

\section{Numerical model}

The numerical model used in this study is a quarterwave thermoacoustic engine, closed on the left side and open to atmospheric pressure on the right side. The resonator is $150 \mathrm{~mm}$ long and $12 \mathrm{~mm}$ wide. Inside the resonator, a stack of parallel plates is placed $30 \mathrm{~mm}$ from the vicinity of the closed end. The parallel plates are 10 $\mathrm{mm}$ long, $0.5 \mathrm{~mm}$ wide, and $0.5 \mathrm{~mm}$ apart. In continuation of our simulation efforts, we have modeled a thermoacoustic refrigerator driven by the thermoacoustic engine. Thermal cooling requires a secondary stack where the pressure amplitude of the wave generated in the driving stack can be attenuated and remove thermal energy from the working fluid. The TAR model extends the previous TAE model by adding a secondary stack just to the right of the driving stack. This model design allows for a complete representation of a heat driven thermoacoustic refrigerator that does not rely on mechanical excitation by oscillating boundary conditions. Unlike most previous models that also simulate thermoacoustic cooling, our model is able to achieve this goal by applying only heat input to the system. The cooling stack $(\mathrm{Sr})$ is represented by 12 parallel plates 10 $\mathrm{mm}$ long, $0.5 \mathrm{~mm}$ wide, and placed $10 \mathrm{~mm}$ from the driving stack (Se). The choice of the values of the geometrical parameters used in the present study is based on previous studies carried out by our group on TAE [24] and on TAR [25], on which these values showed high performance of these systems. The numerical models used in this study are presented in Fig. 4. The geometrical parameters of TAE and TAR and the thermophysical properties of the working fluid are summarized in Table 1.

\subsection{Model space}

The model spaces used to study the influence of plate surface shapes are presented in Fig. 5 . The plate surface forms considered in this work are as follows: (i) flat plate (Fig. 5a): considered as a good reference, particularly for comparison with plates of different shapes. (ii) Plate with rounded ripples (Fig. 5b): the upper horizontal surface of the plates is formed by a series of circular ripples of the same diameter. (iii) Plate with triangular ripples (Fig. 5c): the horizontal surface of the plates is formed by a series of triangular ripples. The choice of these shapes is based on increasing the flow impedance around the plates which could improve the stack performance by constraining gas parcels in the area of the plates.

\subsection{Stack material}

The heat conduction along the stack material and the working fluid in the plate area has an undesirable influence on the efficiency of thermoacoustic devices [26, 27]. There are some important properties for the stack to be suitable for thermoacoustic systems such as the heat capacity of the material constructing the stack cs is required to be higher than the heat capacity of the working fluid and the thermal conductivity $\mathrm{K}_{\mathrm{s}}$ is desired to be smaller as possible to avoid much loss of acoustic power. The material stainless steel is chosen for the present investigation, as it has a low heat conductivity $\left(16 \mathrm{~W} \cdot \mathrm{m}^{-1}\right.$. $\left.\mathrm{K}^{-1}\right)$ and a high capacity $\left(490 \mathrm{~J} \cdot \mathrm{kg}^{-1} \cdot \mathrm{K}^{-1}\right)$.

\subsection{Numerical implementation}

In thermoacoustic systems, the largest scale is the length of the resonator and the smallest scale is the viscous thickness $\delta v$. For a good resolution of the viscous boundary layer, a sufficient number of points of calculation by viscous thickness must be used by refining the area surrounding the parallel plates constituting the stack. The refined and relaxed mesh at different locations used in the CFD simulation is shown in Fig. 6. A mesh sensitivity analysis has been conducted in order to choose the best range of cells for obtaining the optimum results. The acoustic pressure depending on the amount of the cells is presented in Fig. 7. A cell mesh refinement 


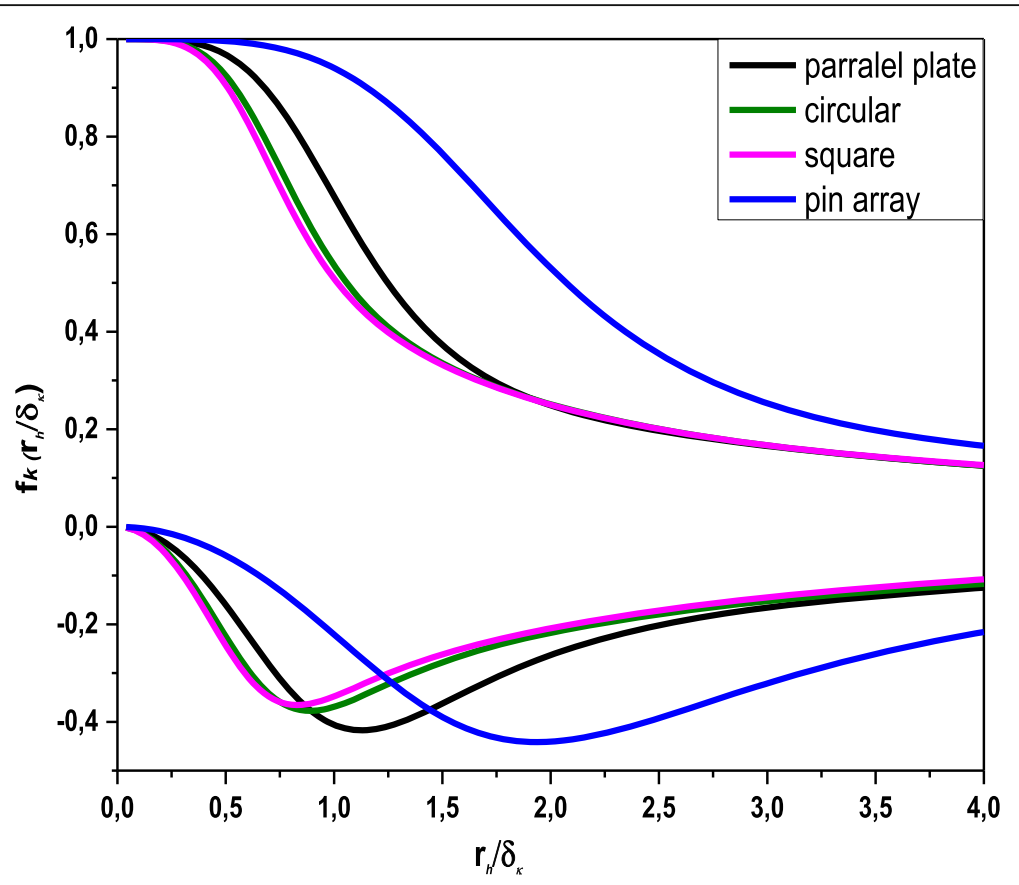

Fig. 3 Rott's functions $\left(f_{k}\right)$ against the ratio of hydraulic radius $\left(r_{h}\right)$ to the thermal penetration depth $\left(\delta_{k}\right)$ for different stack profiles [23]

of 5,000 to 120,000 triangular cells was studied. Noticeably, the mesh refinement affects the acoustic pressure. However, from the 20,000-cell mesh to the most refined, it is observed that the mesh size has less effect on the pressure amplitude. The elements sizes present in the modelspaces were achieved using default "User-controlled mesh" settings within the simulation software
Comsol Multiphysics which is a commercial solver and simulation software used to solve the system of timedependent equations associated with appropriate boundary and initial conditions. The solution of the equations is based on the finite element method; it provides the discretization in the considered domain, and it has appropriate criteria to generate the suited mesh. More

(a) Engine Close End
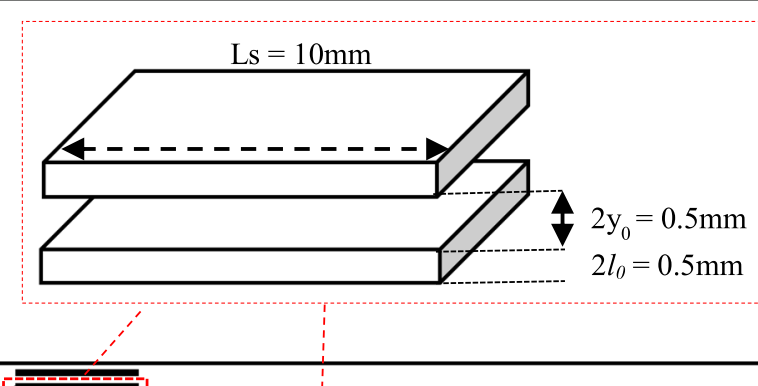

$$
l r=12 \mathrm{~mm}
$$

1

Open End

(b) Refrigerator

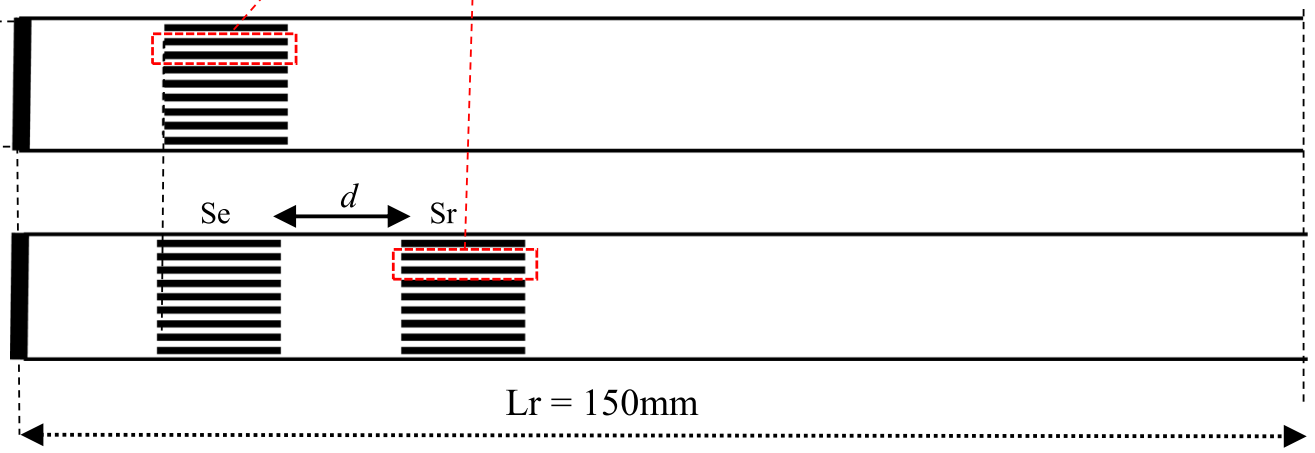

Fig. 4 Geometric modeling of a standing-wave thermoacoustic engine (a) and a thermally driven thermoacoustic refrigerator (b) 
Table 1 Geometrical parameters and thermophysical properties of the working fluid

\begin{tabular}{|c|c|}
\hline Parameter & Value \\
\hline \multicolumn{2}{|l|}{ Resonator } \\
\hline Length (Lr) & $150 \mathrm{~mm}$ \\
\hline Width (Ir) & $12 \mathrm{~mm}$ \\
\hline \multicolumn{2}{|l|}{ Engine stack (Se) } \\
\hline Number of plates & 12 \\
\hline Plate length (Lse) & $10 \mathrm{~mm}$ \\
\hline Plate thickness $\left(2 \mathrm{l}_{0}\right)$ & $0.5 \mathrm{~mm}$ \\
\hline Plate spacing $\left(2 \mathrm{y}_{0}\right)$ & $0.5 \mathrm{~mm}$ \\
\hline Position of hot end (xh) & $10 \mathrm{~mm}$ \\
\hline Position of cold end (xc) & $20 \mathrm{~mm}$ \\
\hline \multicolumn{2}{|l|}{ Refrigeration stack (Sr) } \\
\hline Number of plates & 12 \\
\hline Plate length (Lsr) & $10 \mathrm{~mm}$ \\
\hline Plate thickness $\left(2 \mathrm{I}_{0}\right)$ & $0.5 \mathrm{~mm}$ \\
\hline Plate spacing $\left(2 \mathrm{y}_{0}\right)$ & $0.5 \mathrm{~mm}$ \\
\hline Stack distance (d) & $10 \mathrm{~mm}$ \\
\hline \multicolumn{2}{|l|}{ Working fluid } \\
\hline Type & Air (Ideal gas) \\
\hline Density & $1.1768 \mathrm{~kg} / \mathrm{m}^{3}$ \\
\hline Specific heat ratio & 1.4 \\
\hline Specific gas constant & $287 \mathrm{~J} /(\mathrm{kg} . \mathrm{K})$ \\
\hline Speed of sound c & $348 \mathrm{~m} / \mathrm{s}$ \\
\hline Thermal conductivity $\mathrm{K}$ & $0.0263 \mathrm{~W} /(\mathrm{m} . \mathrm{K})$ \\
\hline Isobaric specific heat $\mathrm{cp}$ & $1006 \mathrm{~J} /(\mathrm{kg} . \mathrm{K})$ \\
\hline
\end{tabular}

details of Comsol Multiphysics software are presented in reference [28].

\subsection{Steady and transient simulation}

In the present work, the surfaces of the resonator are used as adiabatic walls, except at the open end, where a temperature of $300 \mathrm{~K}$ has been imposed. Temperatures of $T_{\mathrm{c}}=900 \mathrm{~K}$ and $T_{\mathrm{c}}=300 \mathrm{~K}$ are applied to the hot and cold ends of the engine stack, respectively. An initial pressure perturbation of $10 \mathrm{~Pa}$ was introduced at the closed end of the resonator to create non-zero values for pressure and velocity throughout the system. The initial pressure perturbation $(10 \mathrm{~Pa})$ and the temperature imposed on the hot side of the engine stack (900 K) are chosen after a sensitivity study (Section 1.6).

\subsubsection{Steady simulation}

The numerical instabilities are not sufficient to cause the thermoacoustic effect, so a small pressure disturbance must be introduced into the system. Thus, a pressure of $10 \mathrm{~Pa}$ gauge is applied at the closed end of the resonator. This results in non-zero values for the pressure and velocity throughout the system, as well as the initialization of the temperature distribution in the stack area. Figures 8 and 9 show the contours and distribution of pressure, temperature, and velocity along the system during the steady state simulation applied for the thermoacoustic engine. This initial condition shows a high pressure in the closed end and a pressure drop across the stack. With this initial condition, it was possible to reproduce the thermoacoustic effect indicated by strong pressure oscillations in the resonator. It can be seen that the pressure drops sharply in the area of the stack, from the

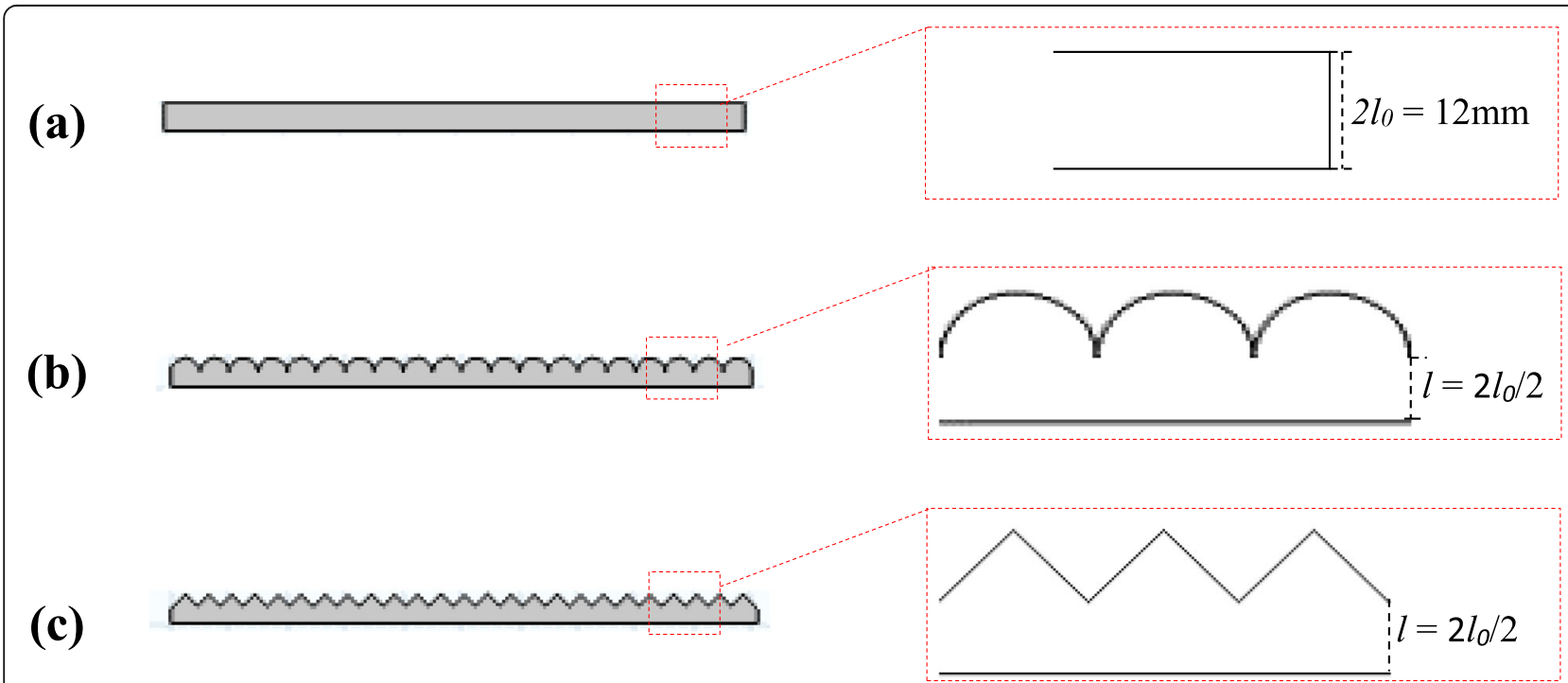

Fig. 5 Sketches of model spaces used for studying different stack plate shapes: a flat plate, b plate with rounded ripples, and c plate with triangular ripples. All model spaces presented are otherwise same to that showed in Fig. 4 


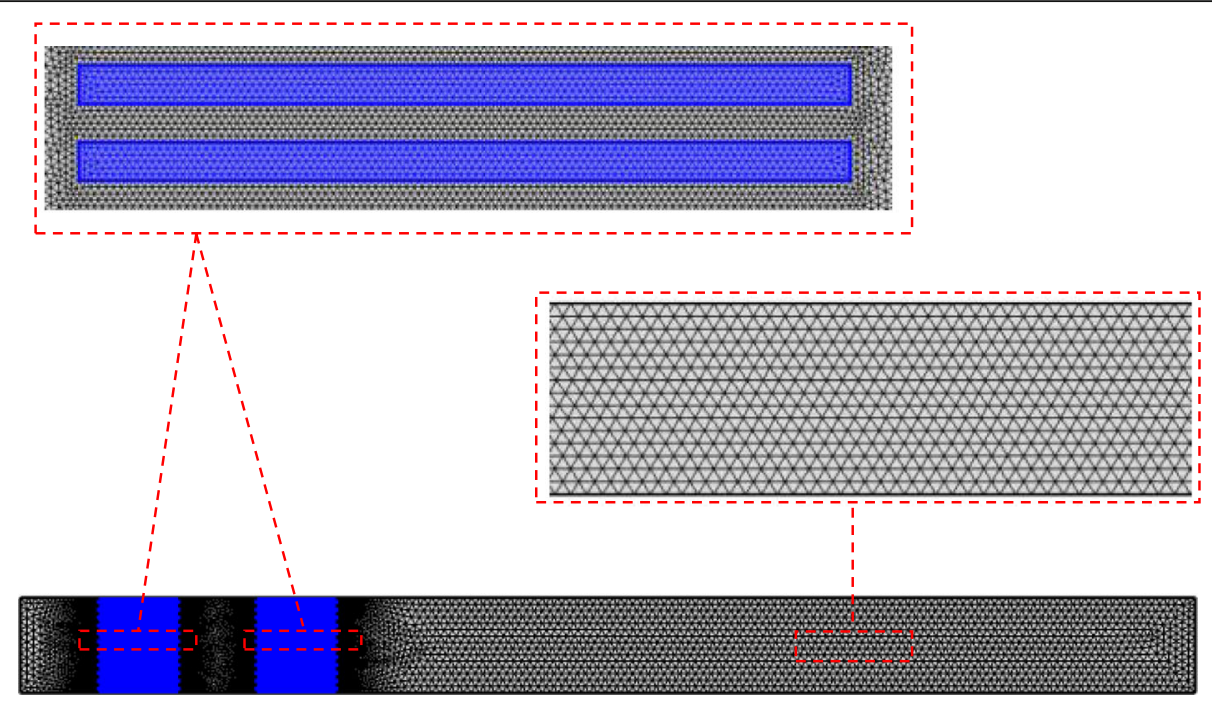

Fig. 6 Refined and relaxed mesh used for the CFD simulation

assumed value of $10 \mathrm{~Pa}$ to the value of $0 \mathrm{~Pa}$. On the other hand, the velocity increases at the stack, the highest value found being about $1.29 \mathrm{~m} . \mathrm{s}-1$ recorded at the position $x=31 \mathrm{~mm}$.

\subsubsection{Transient simulation}

For the transient simulation, the time steps have been chosen to be constant. The time step is chosen to be $10^{-5} \mathrm{~s}$. This value was large enough to allow convergence for each time step and small enough to allow the time steps to advance rapidly. The simulation successfully reproduces the amplification of the acoustic pressure waves maintained in the resonator. Figure 10 shows the acoustic oscillations achieved from the transition of the amplification to a steady-state. The final amplitude of the acoustic pressure is about $23.3 \mathrm{kPa}$, which corresponds to a sound pressure level (SPL) of:

$$
\begin{aligned}
& S P L=20 \log _{10}\left(\frac{P_{a c}}{P_{r e f}}\right)[d B] \\
& S P L=20 \log _{10}\left(\frac{23300}{2.10^{-5}}\right)=181.3 d B
\end{aligned}
$$

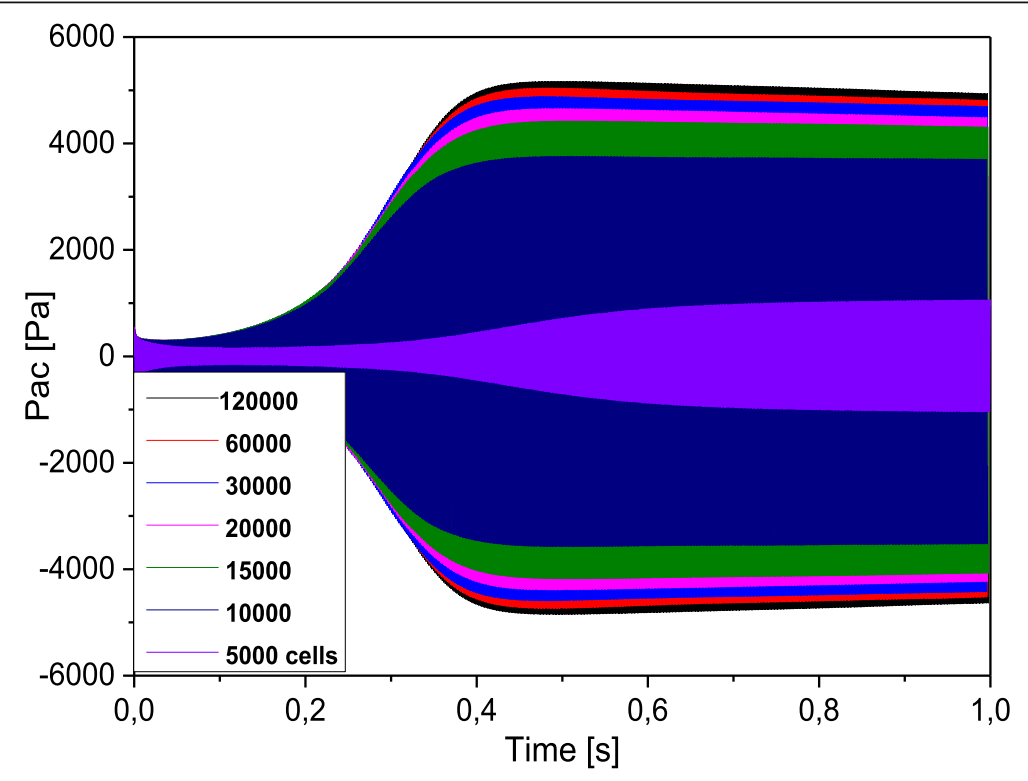

Fig. 7 Acoustic pressure amplitude achieved in TAE depending on grid properties 
where $P_{\mathrm{ac}}$ is the acoustic pressure, and $P_{\text {ref }}$ is the standard reference of 20 micropascals in the air (the pressure of the smallest sound we can hear Pref = $\left.2.10^{-5} \mathrm{~Pa}\right)$. It is worth mentioning that the highest sound pressure level reported in the literature is $190 \mathrm{~dB}$ [29], achieved in an advanced thermoacoustic engine. These values are very high considering the simple design of the engine. However, this simulation did not take into

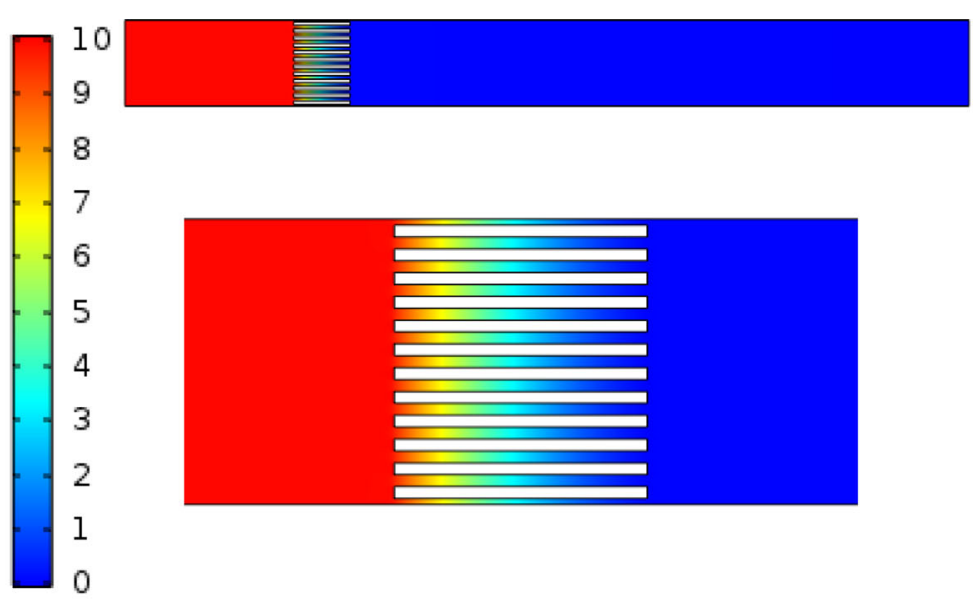

(a) Pressure $[\mathrm{Pa}]$

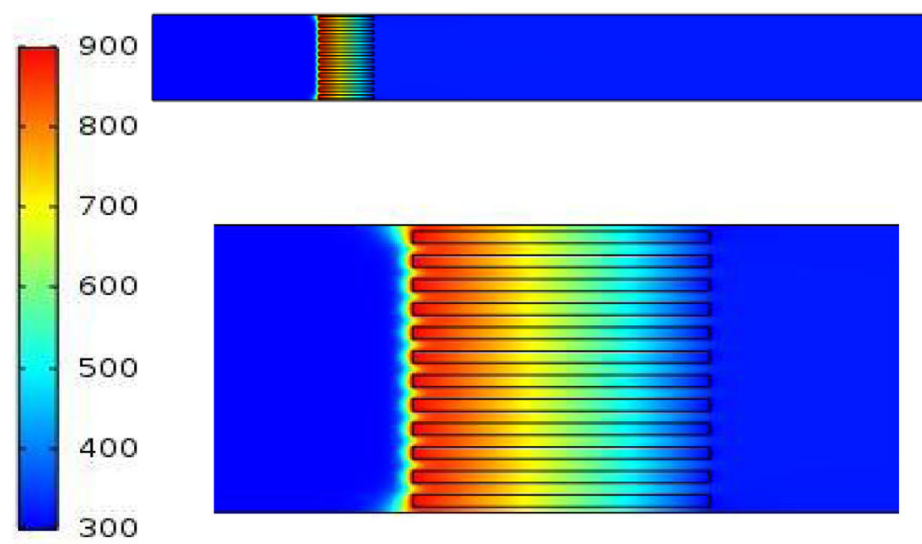

(b) Temperature $[\mathrm{K}]$

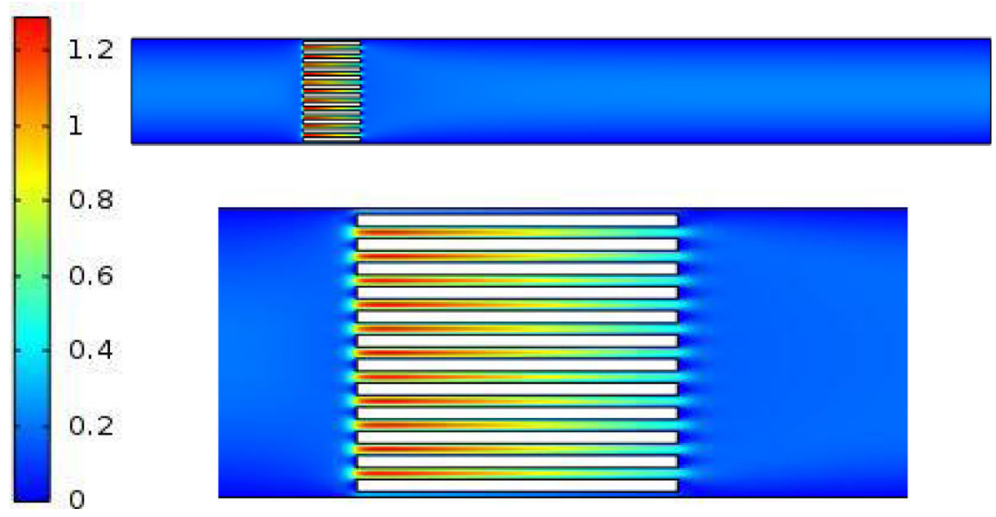

(c) Velocity $\left[\mathrm{m} \cdot \mathrm{s}^{-1}\right]$

Fig. 8 Initialization of the pressure perturbation. Distribution of a pressure, $\mathbf{b}$ temperature, and $\mathbf{c}$ velocity along the resonator and at the stack area 


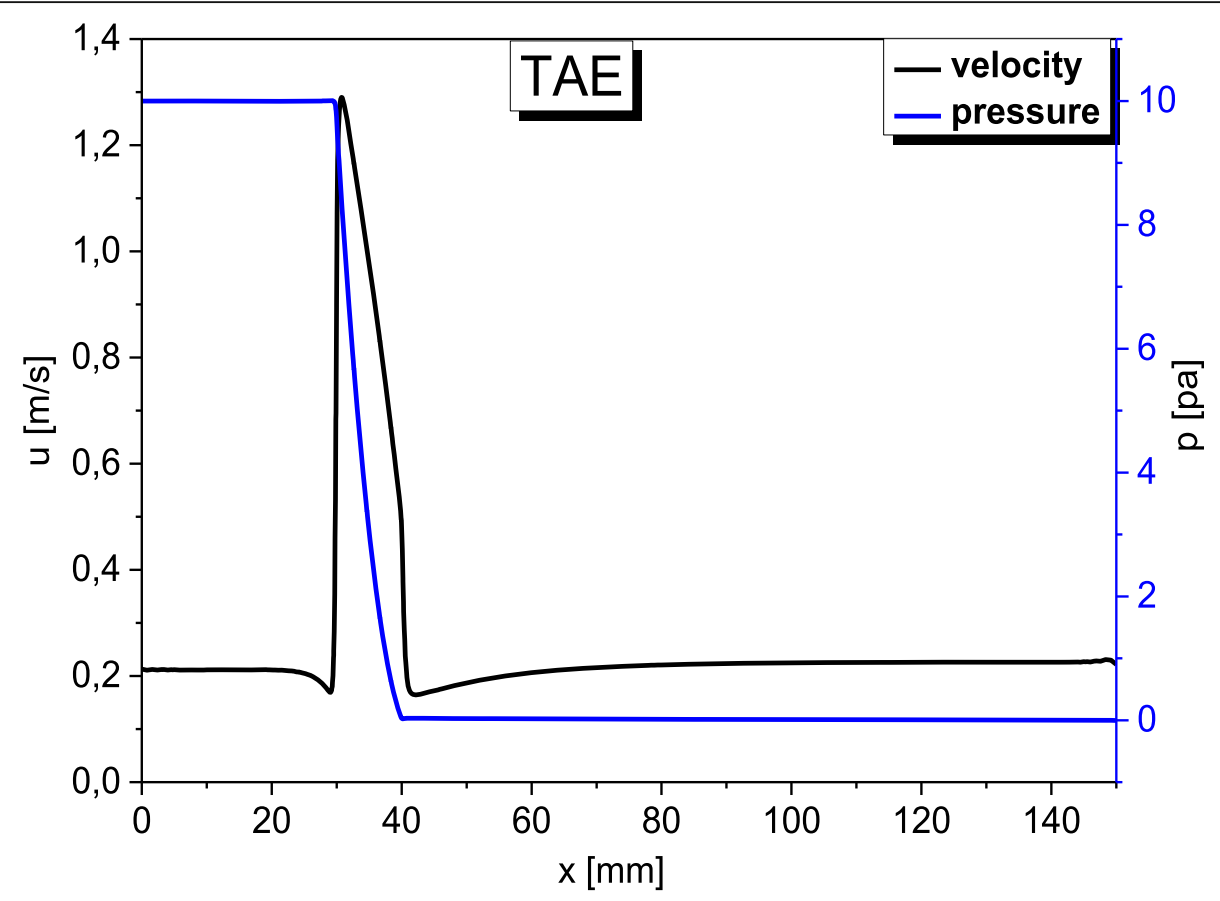

Fig. 9 PRESSURE and velocity reached at the steady state along the resonator of TAE

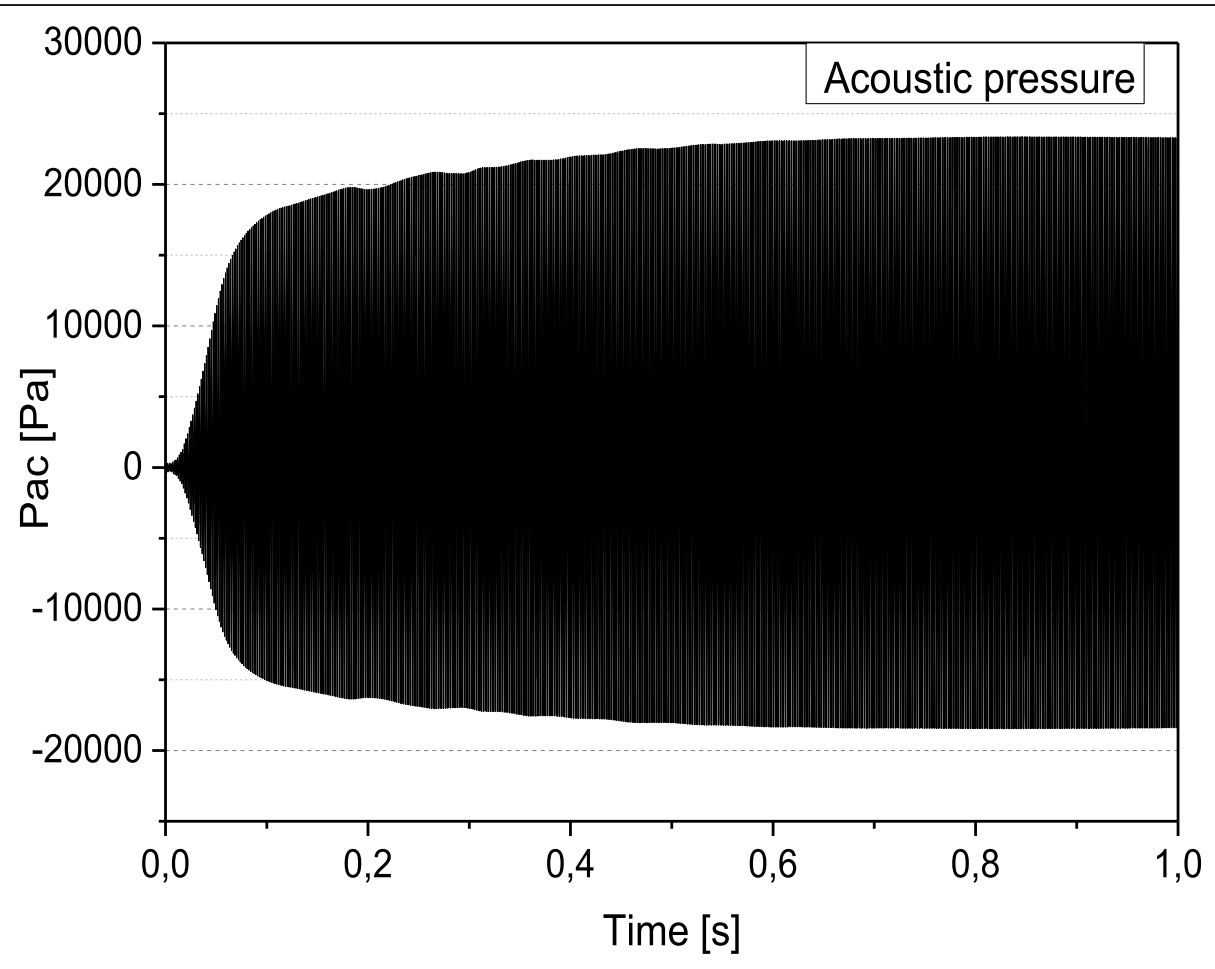

Fig. 10 Acoustic oscillations developed by TAE at transient simulation 
account loss mechanisms (such as heat flow through the walls). It is therefore not surprising to obtain high sound pressure levels. During the steady state, energy is dissipated through turbulence generation and through the open end.

\subsection{Model validation}

To ensure that our model is correct, a comparative study is carried out to determine the accuracy of the numerical model adopted in this study with the results found by Zink et al. [30], who developed a numerical model of a standing wave TAE with a stack of parallel plates; a temperature gradient and a heat transfer coefficient were applied to the horizontal surfaces. This modeling does not require the assignment of plate material. The same accurate properties and characteristics were applied again for our model. After conducting the simulation of the model from the transition phase of the initial pressure perturbation to the steady-state and by applying fast Fourier transform (FFT) analysis to the sound pressure oscillations, the results show that the acoustic pressure and the frequency are about $4694 \mathrm{~Pa}$ and $584 \mathrm{~Hz}$, respectively. This frequency corresponds with the theoretical operating frequency of a thermoacoustic engine with a length of $0.15 \mathrm{~m}$. The comparison of the acoustic pressure and frequency of oscillations obtained in this study with the results found by [30] shows a difference of $1.35 \%$ and $3.83 \%$ in frequency and acoustic pressure, which is a tolerate difference for the results.

\subsection{Sensitivity study}

The initial pressure disturbance $(10 \mathrm{~Pa})$ and the hot temperature imposed at the hot side of the engine stack $(900 \mathrm{~K})$ are chosen after a sensitivity study on the influence of these parameters on the thermoacoustic effect. Figure 11 shows the acoustic pressure behavior resulting from six different initial pressure perturbations. The shapes of all curves are similar, but the amplitude of these initial oscillations is different in each sample. It can be seen that the smallest value introduced $(1 \mathrm{~Pa})$ gives a very small final amplitude, which is not sufficient to obtain a good representation of the thermoacoustic effect. However, when the initial perturbation is increased to $5 \mathrm{~Pa}$ and above, thermoacoustic oscillations are obtained. Each value shows an initial decrease in pressure amplitude at the beginning of the transient simulation. The resulting pressure amplitudes at the steady state are shown in Table 2. The final amplitudes for $5 \mathrm{~Pa}$ and $10 \mathrm{~Pa}$ are very similar. A further increase shows a decrease in the final amplitude. Since the initial perturbation of $10 \mathrm{~Pa}$ gives better oscillations, it is therefore chosen as the initial perturbation for this study. The temperature of the hot end of the engine stack is chosen to be $900 \mathrm{~K}$. In order to show the influence of this choice, additional simulations were carried out using different temperature ranges. For the temperature applied to the hot side of the driven stack, we applied different temperatures (from 600 to $1000 \mathrm{~K}$ ) to the hot side of the stack while keeping the ambient side at a constant temperature of $300 \mathrm{~K}$. Figure 12 shows the process of acoustic wave amplification as a function of time against the hot side temperature. It is clear that the amplitude of the acoustic pressure increases as the temperature gradient between the ends of the stack increases. The acoustic pressure amplitude for the $700-300 \mathrm{~K}$ temperature gradient is equal approximately to $38 \mathrm{~Pa}$ and exceeds $5600 \mathrm{~Pa}$ for the $1000-300 \mathrm{~K}$ gradient. However, for $T_{\mathrm{h}}=600 \mathrm{~K}$, the thermoacoustic engine could not induce the thermoacoustic effect. The final amplitudes for $900 \mathrm{~K}$ and $1000 \mathrm{~K}$ are very close. For all other simulations, the hot-side temperature was chosen to be $900 \mathrm{~K}$.

\section{Results and discussion}

This part is shared into two sections. First, the simulation results obtained for the thermoacoustic engine with modified stack plates where the time-dependent evolution of the acoustic oscillations achieved from the initial disturbance to the steady state and the acoustic pressure amplitude are shown and compared by graphs. In the second section, results of each plate shape obtained for the thermally driven thermoacoustic refrigerator will be compared based on effective temperature gradient along with the refrigeration stack and the average temperature reached at the cold side of the refrigeration stack.

\subsection{Thermoacoustic engine}

The acoustic pressure amplitude is considered a key parameter for estimating the strength of thermoacoustic oscillations. This parameter is of excessive significance to a thermoacoustic engine to drive any thermoacoustic system. The processes of evolution of the self-excited thermoacoustic oscillations from the initial disturbance to the steady state, the acoustic pressure amplitude at initial transition, the acoustic pressure amplitude at the steady state and the pressure amplitude along the engine for all stack plate surface shapes considered are shown in Figs. 13, 14a, b, and c, respectively. The amplitude of the acoustic pressure obtained in the thermoacoustic engine is taken at the closed end of the resonator which represents the pressure antinode. A time step of $10^{-5}$ including 100 thousand time steps $(1 \mathrm{~s})$ is chosen to perform the simulations. The phenomenon of interaction between fluid and solid wall responsible for the thermoacoustic effect involves complex processes and requires precise modeling. However, a conceptual approach simplified based on CFD analysis allows understanding the mechanisms. In self-exciting thermoacoustic machines, 


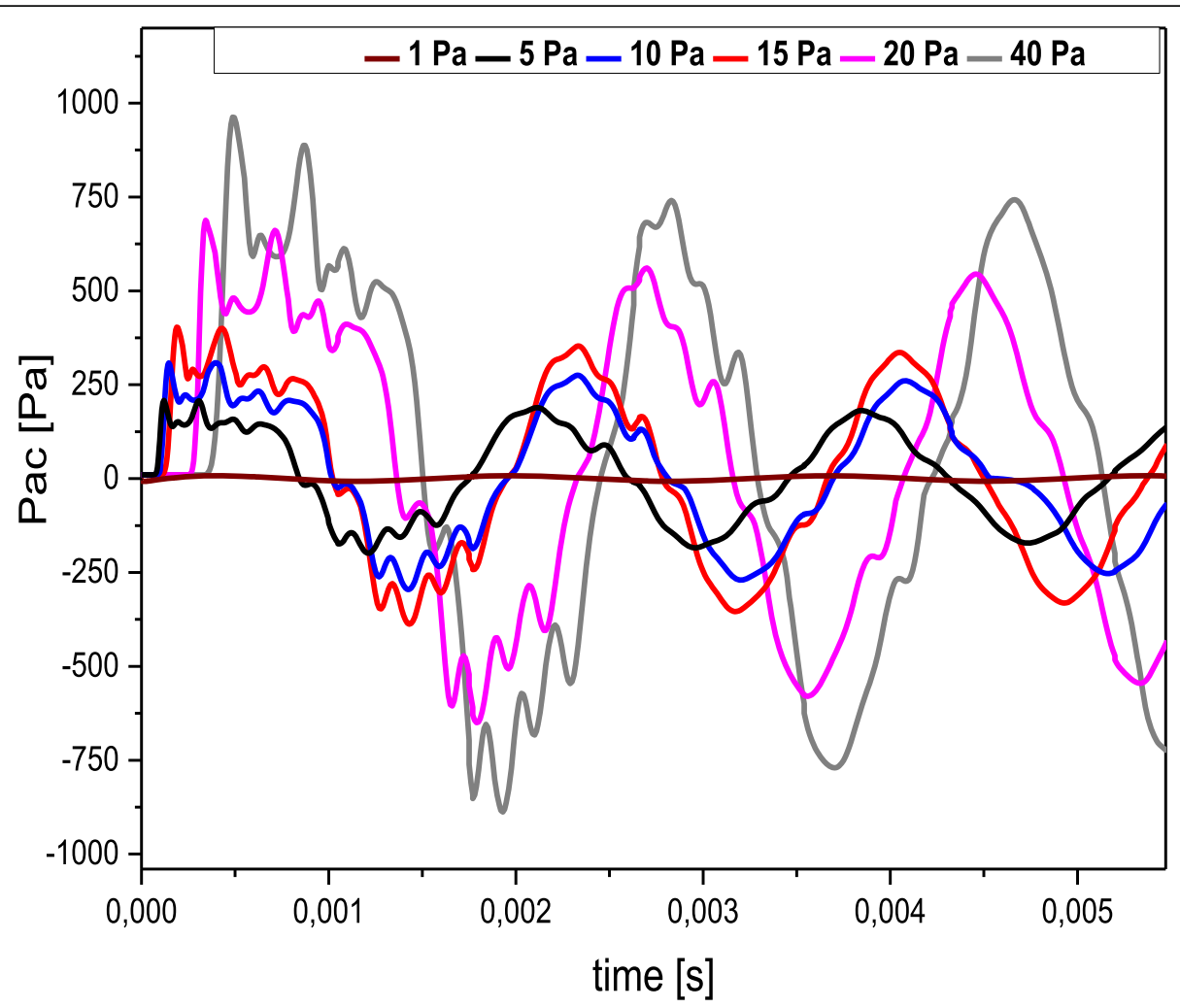

Fig. 11 Pressure oscillations with different initial pressures

it is assumed that the oscillatory movements of the working fluid are maintained, which leads to a conversion of the thermal energy provided by the hot heat exchanger into an acoustic power generated at the chimney and amplified by the resonator. By dividing it into three parts, the self-excitation process can be described as follows:

First, the hot end of the engine stack is continuously heated by applying a high temperature of $900 \mathrm{~K}$ while the temperature of the cold end is fixed at a constant temperature of $300 \mathrm{~K}$. Thus, a strong temperature gradient between the stack ends is created. The gas perturbations generated by the thermal gradient imposed on the ends of the plates accelerate progressively and rapidly. These perturbations are necessary to overcome the thermal dissipation near the stack so that the acoustic wave can reach the entire resonator during its propagation process. Finally, this acoustic wave occurs continuously and ends up being amplified to a high amplitude along

Table 2 Pressure amplitudes for different initial pressure disturbances

\begin{tabular}{llllll}
\hline $\mathbf{P}_{\mathbf{i}}[\mathrm{Pa}]$ & $\mathbf{5}$ & $\mathbf{1 0}$ & $\mathbf{1 5}$ & $\mathbf{2 0}$ & $\mathbf{4 0}$ \\
\hline $\mathrm{P}_{\mathrm{ac}}[\mathrm{Pa}]$ & 4632 & 4695 & 4452 & 4213 & 3671 \\
\hline
\end{tabular}

the resonator. The evolution process of the acoustic pressure generated in the thermoacoustic engine for all the profiles studied can be divided into three parts: first, the instability of the process takes place in the first milliseconds as presented in Fig. 14a. This phase occurs when the thermal gradient along the stack reaches the gradient imposed at the ends of the plates. After this phase, the different viscous and thermal energy losses to the surrounding medium approach equilibrium which leads to a continuous increase of the pressure amplitude. Last phase, the progress of the acoustic wave produced by conversion thermal energy into acoustic energy reaches the steady state after heating the stack for approximately $43,000,80,000$, and 90,000 iterations for flat plates, for plates with rounded ripples and plates with triangular ripples, respectively. While Fig. 14b shows the acoustic pressure, amplitude reached at the steady state. The plates with triangular and rounded ripples are noticeable to be more effective in increasing the pressure amplitude than the flat plates with a pressure amplitude of approx. $25.8 \mathrm{kPa}$ and $24.5 \mathrm{kPa}$ respectively. The triangular profile performs noticeably inversely from the other profiles; it is more effective with a benefit of $10 \%$ than using a flat plate, followed by rounded ripples with $5.2 \%$ of the increase in pressure amplitude. Obviously, the plate shape affects the excitation of the 


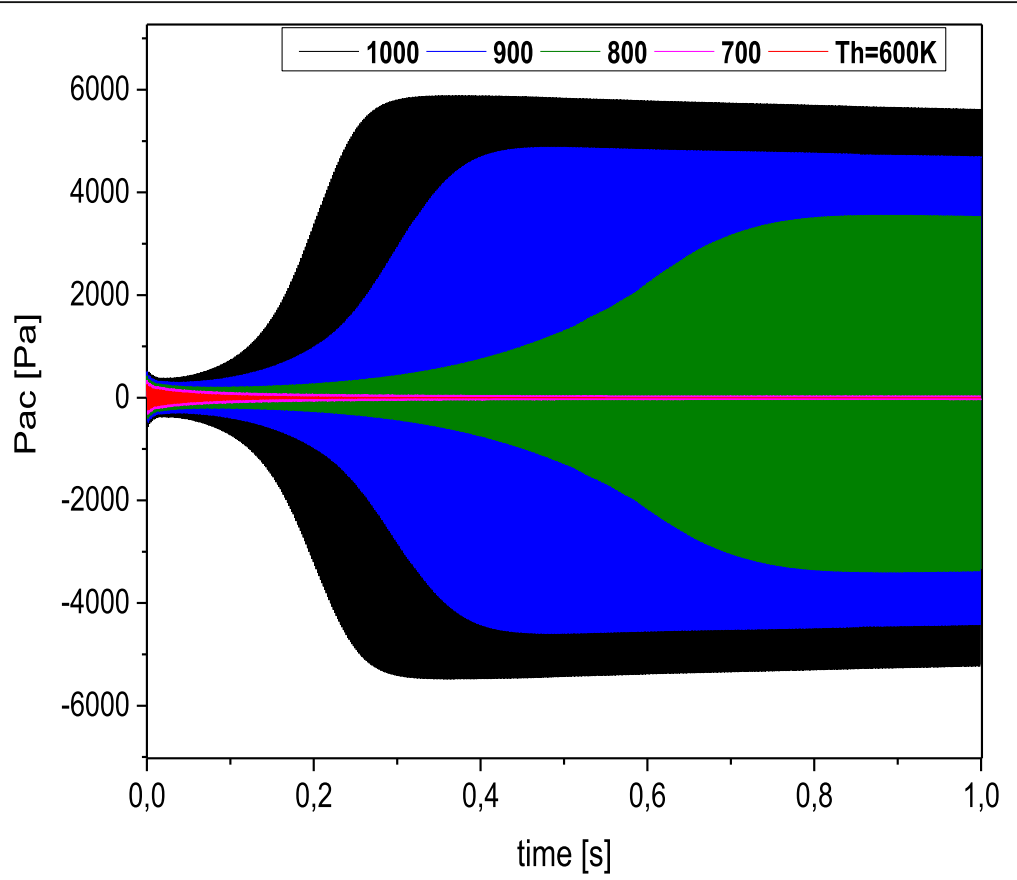

Fig. 12 Pressure oscillations with different hot temperatures

thermoacoustic engine significantly It was expected that this result would also be achieved since plate ripples present curved surfaces to reduce flow impedance between the hot and the cold sides of the stack. As a continuation of our efforts to study the effect of plate surface shape on the thermoacoustic effect in a thermoacoustic engine, we conducted a study with different depths of corrugations. Figure 15 presents the acoustic pressure amplitude reached at the steady state for rounded and triangular ripples with different depths of ripples (the ratio $\left.l / l_{p}\right)$. The results show that the plates with a high ratio $1 / 1 \mathrm{p}$ are more effective in increasing the pressure amplitude compared to the plates with lesser $l / l_{p}$.

Starting with the plates with rounded ripples, the pressure amplitude increases by approx. $23.4 \mathrm{kPa}$ up to 24.6 $\mathrm{kPa}$ by increasing the ratio $1 / \mathrm{l}_{\mathrm{p}}$ from $1 / 4$ to $3 / 4$, respectively with a benefit of $4.8 \%$. The same scenario is noticed for plates with triangular ripples with different ratios; the pressure amplitude increases from $23.7 \mathrm{kPa}$ to 26.7 $\mathrm{kPa}$ with an increase of $1 / \mathrm{l}_{\mathrm{p}}$ from $1 / 4$ to $3 / 4$ respectively with a benefit of $11.2 \%$. Clearly, the depth of ripples affects the energy conversion significantly. It was expected that this outcome would also be obtained since plate ripples present corrugated surfaces to reduce flow impedance among the hot and the cold sides of the engine stack. To better understand the effect plates with corrugated surfaces on the energy conversion in the thermoacoustic engine, we go to the simple linear expressions of the normalized acoustic energy produced by the engine stack $\left(W_{n}\right)$ expressed by Equation (13) in which involved the stack porosity expressed by Eq. (19). By keeping the gap of plates constant, using plates with depth ripples will increase the stack porosity. This clarifies why the acoustic pressure amplitude differs from one shape to another; this is observed at most for the stack of plates with triangular ripples where the pressure is highest. To better show the effect of BR on the acoustic power generated in the thermoacoustic engine, we plot the normalized acoustic power produced by the stack engine against the normalized stack length for different blockage ratios as presented in Fig. 16. It shows that for different given values of BR the produced acoustic energy has an optimum value, the best performance presented is shown for $\mathrm{BR}=0.5$ which corresponds to a stack of which the thickness plate is equal to the gap between two plates.

\subsection{Thermoacoustically driven thermoacoustic refrigerator} Unlike engines, refrigerators extract a quantity of heat from a cold environment to a warm environment, from the supply of mechanical power. One thing in common with engines is that there are refrigerators that work with standing waves and others with traveling waves. The main difference between the two types of refrigerators comes from the source used: in the first case, it is a loudspeaker of high impedance, piezoelectric, etc., which excites a resonance specific to the system, while in the second case, the pressure wave of large amplitude is obtained by pistons developing a large stroke. In these machines, the efficiency is limited by the losses by thermal 

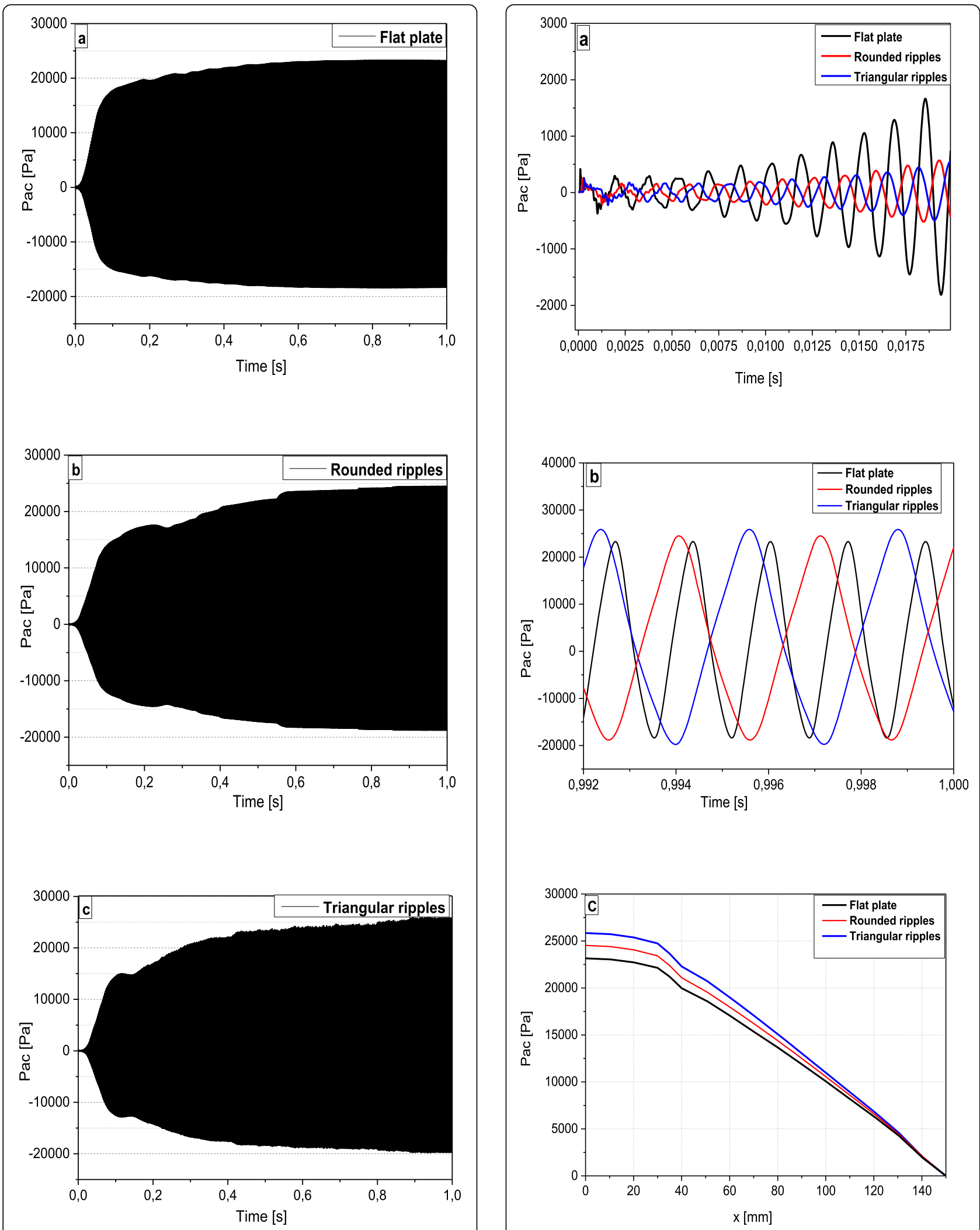

Fig. 13 Acoustic pressure oscillations for different plate shapes studied

Fig. 14 Acoustic pressure amplitude at the first disturbance (a), at the steady state (b), and depending on the length of TAE (c) 

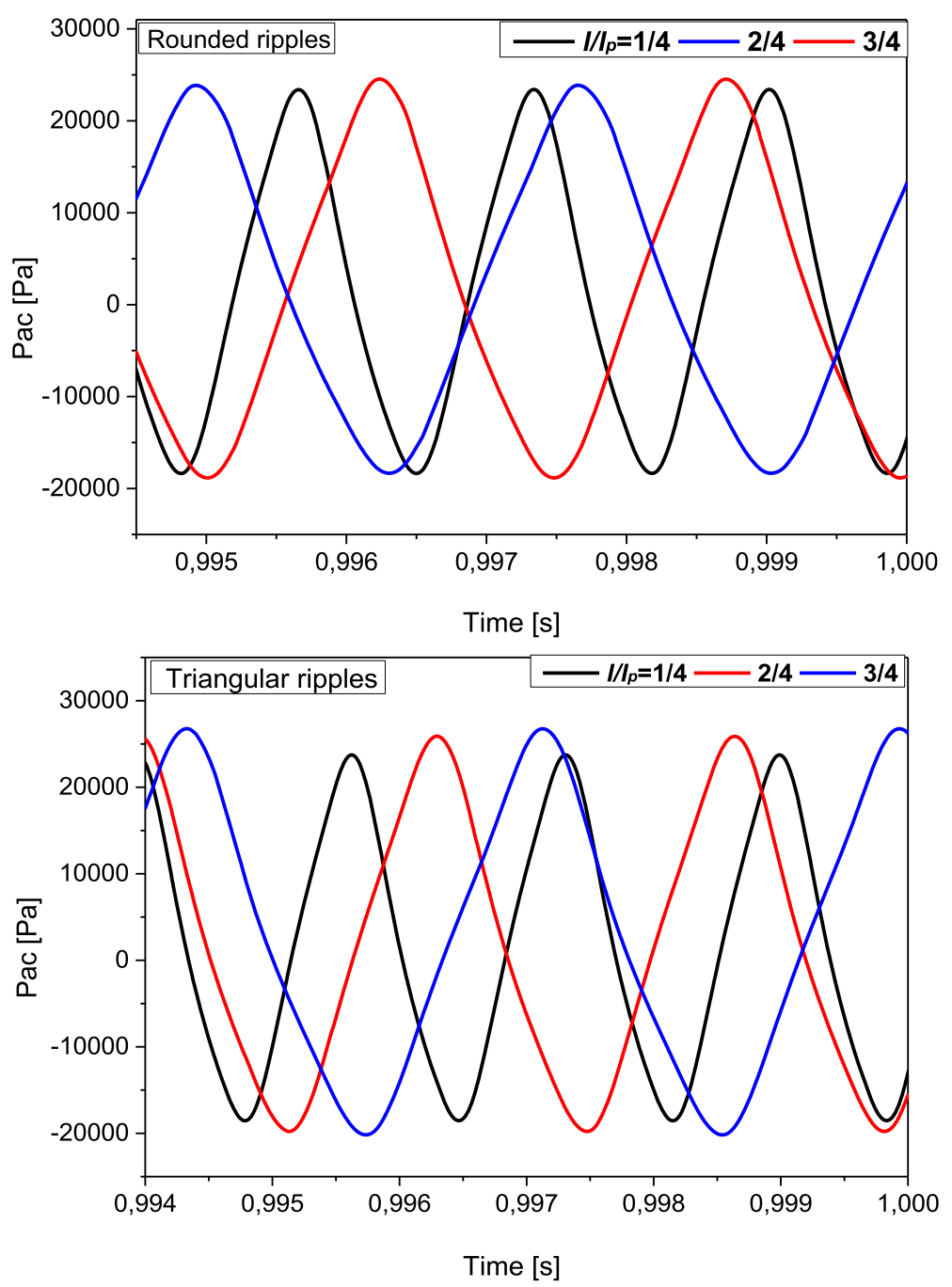

Fig. 15 Acoustic pressure amplitude at the steady state for rounded and triangular ripples with different depth

conduction in the axial direction, viscous friction, or even by thermal relaxation. A major condition for the efficient operation of a thermoacoustic refrigerator is to have a sufficiently powerful acoustic source, which current loudspeaker technology or piezoelectric sources have difficulty in achieving. Given the relative simplicity of implementation of the thermoacoustic process, it is possible to envisage generating the acoustic wave by a thermoacoustic engine, by coupling a thermoacoustic wave generation system and a thermoacoustic heat pumping system, where a source of hot heat produces an acoustic wave which in turn is used to generate cold. The most widely used system is the thermally driven thermoacoustic refrigerator shown schematically in Fig. $4 \mathrm{~b}$ : it offers good performance and allows very low temperatures to be reached, perhaps the most famous system using such a coupling is the Beer Cooler [26]. We consider here the case of a thermally driven thermoacoustic refrigerator driven by a thermoacoustic engine. When the parallel plates of the refrigeration stack, initially in equilibrium with the surrounding environment, are subjected to a standing wave, a flow of thermoacoustic energy occurs in the fluid parallel to the plates. This flow is directed towards the nearest pressure node. Under the effect of this flow, a mean temperature gradient $\nabla \mathrm{T}_{\mathrm{m}}$ will gradually be created in the plate extremities and the fluid surrounding these plates. In the absence of losses, this gradient would increase to the limit value of the critical gradient. However, in reality, the value of $\nabla \mathrm{T}_{\mathrm{m}}$ is limited by the losses and particularly by the heat flow by thermal conduction which opposes the thermoacoustic flow. Thus, it is possible to evaluate the temperature gradient or even the temperature difference $\Delta \mathrm{T}=\mathrm{Ls}_{\mathrm{T}} \mathrm{T}_{\mathrm{m}}$ between the ends of the stack, when the equilibrium state is reached. In this section, we are again interested in the temperature difference $\Delta \mathrm{T}$ 


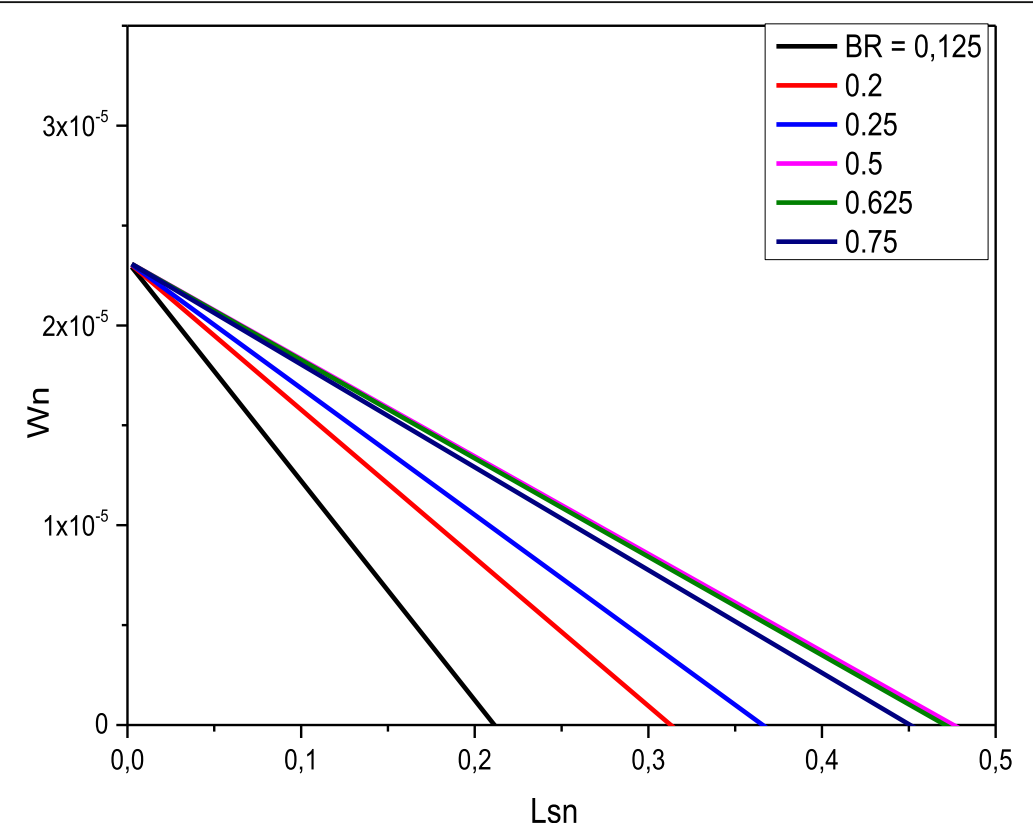

Fig. 16 Normalized acoustic power produced in the engine stack versus normalized stack length for different BR

created between the ends of the refrigeration stack in a thermally driven thermoacoustic refrigerator. In particular, comparisons are made with linear theory. The interest of this comparison is various. First, in the absence of heat exchangers, the average temperature is not forced at any point in the domain. Thus, the correct evaluation of the temperature difference is an important test for our simulation tool. In addition, the comparison makes it possible to test the influence of plate shapes on the temperature gradient between the ends of the stack and its limits. In particular, the comparison allows us to test the effectiveness of the ripples applied to the surface of the plates. Figures 17 and 18 present the temperature evolution at the cold (Tc) and the hot side (Th) and the evolution of the average temperature of refrigeration stack, respectively, achieved for a stack with flat plates, rounded ripples, and triangular ripples. Figures show that the stack made of flat plates is more effective by producing a higher temperature difference along the refrigeration stack $(\Delta \mathrm{T} \approx 16.5 \mathrm{~K})$ than plates with rounded and triangular ripples with $\Delta \mathrm{T} \approx 7.8 \mathrm{~K}$ and $5.7 \mathrm{~K}$, respectively. Replacing flat plates with rounded ripples, $\Delta \mathrm{T}$ undergoes a reduction of up to $52 \%$, and up to $65 \%$ if using triangular ripples. By keeping the plates gap constant and using plates with ripples, the stack porosity increases, expressed by Eq. (19). This justifies why the temperature difference differs from one shape to another; this is observed at most for the stack with triangular ripples where $\Delta \mathrm{T}$ is lowest. To better understand the effect of BR on the cooling power generated by the refrigeration stack, Eq. (14), we plot the normalized cooling power $\left(Q_{\mathrm{cn}}\right)$ behavior against normalized stack length for different blockage ratios (Fig. 19). It is clear that by increasing the blockage ratio the performance of the stack decreases. The best performance is shown for $\mathrm{BR}=0.2$

\section{Conclusion}

In this work, a two-dimensional numerical analysis based on CFD simulation carried out using the software COMSOL Multiphysics is considered to investigate the performance of thermoacoustic couples using a variety of stack plate surface profiles. The comparative performance of three plate shapes noted, flat plate, plate with rounded, and triangular ripples have been estimated. The efficiency of the thermoacoustic engine is examined in terms of the generated acoustic pressure, and the performance of the thermoacoustic refrigerator is measured in terms of the temperature difference along the refrigeration stack. The results show that the plates with triangular and the rounded ripples are noticeable to be more effectively in increasing the acoustic pressure amplitude than the flat plates. The triangular shape performs noticeably more effective with a benefit of $10 \%$ than using a flat plate, followed by rounded ripples with $5.2 \%$ of the increase in acoustic pressure amplitude. The results show that the plates with a high ratio $l / l_{p}$ (the ratio of the depth of ripples to the thickness of the plate) are more effective in increasing the pressure amplitude compared to the plates with lesser $l / l_{p}$. By increasing the ratio $l / l_{p}$ from $1 / 4$ up to $3 / 4$, the acoustic pressure amplitude increases by $4.8 \%$ by using a stack of plates 

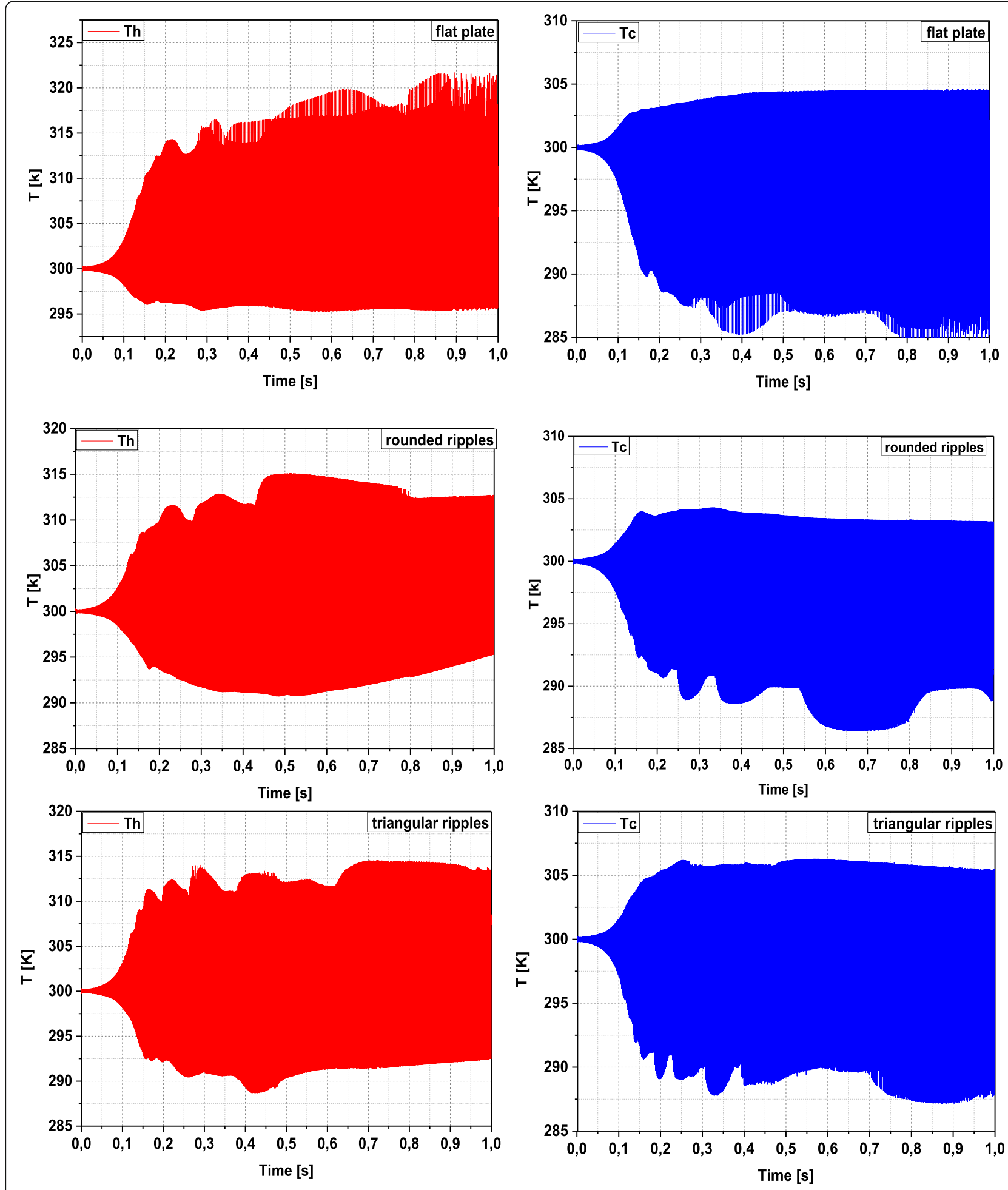

Fig. 17 Temperature evolution at the hot and the cold end of refrigeration stack for all cases studied

with rounded ripples and by $11.2 \%$ for triangular ripples. Unlike for the thermoacoustic refrigerator, the stack made of flat plates can produce a higher temperature difference $(\Delta \mathrm{T})$ between stack ends. the stack made of flat plates are able to give a higher temperature difference between stack ends, $\Delta \mathrm{T} \approx 16.5 \mathrm{~K}$, than plates with rounded and triangular ripples where $\Delta \mathrm{T} \approx 7.8 \mathrm{~K}$ and $5.7 \mathrm{~K}$, respectively. Shifting from a flat 


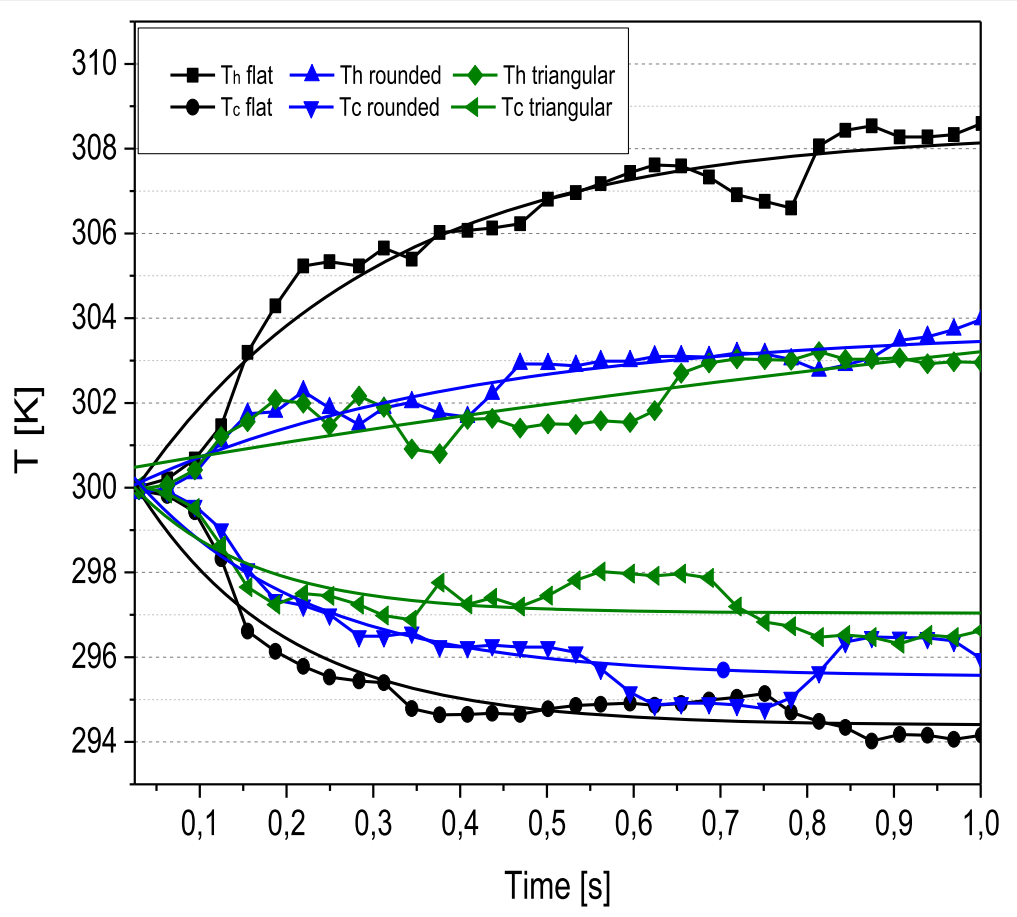

Fig. 18 Average Temperature evolution at the hot and the cold end of refrigeration stack for all cases studied

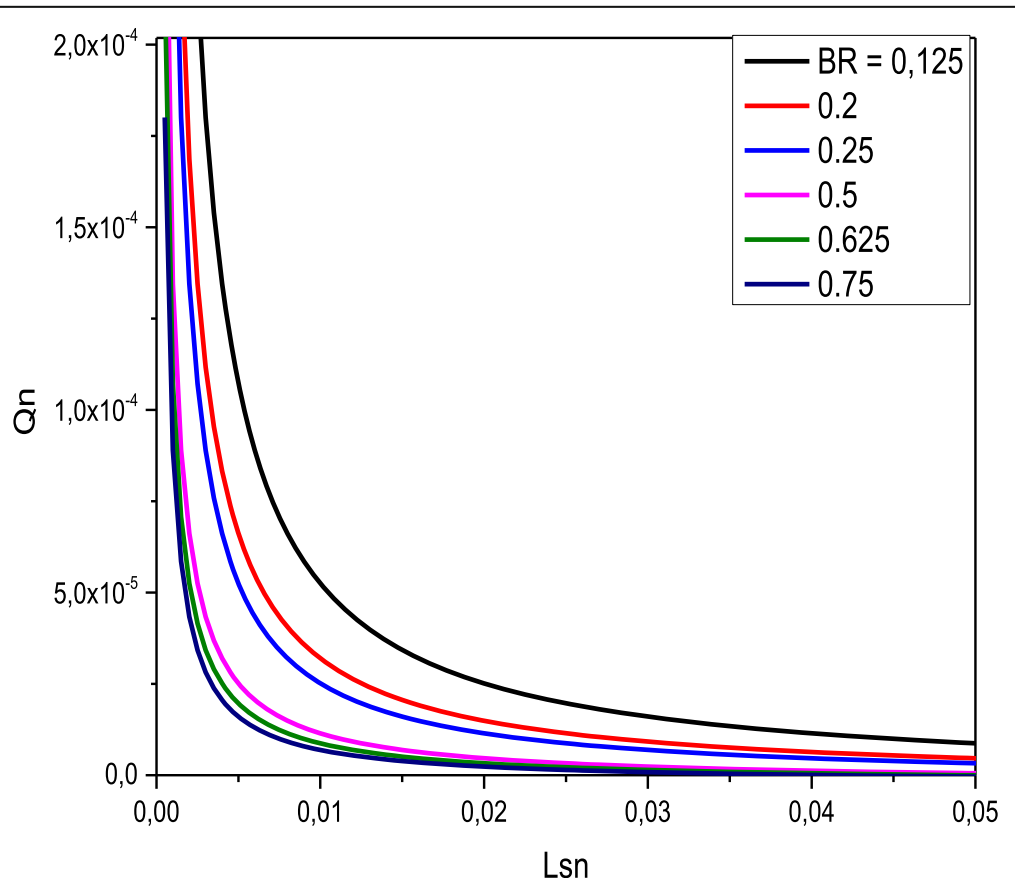

Fig. 19 Normalized cooling power versus normalized stack length for different blockage ratios 
plate to a rounded surface, $\Delta \mathrm{T}$ undergoes a reduction of up to $52 \%$, and up to $65 \%$ if using plates with triangular ripples. These results are explained by the effect of increasing the stack porosity on the acoustic energy produced in the engine and the cooling power generated by the thermoacoustic refrigerator. By using plates with depth ripples, the cross-sectional area of the gas will increase so as the stack porosity.

\section{Nomenclature}

a: Sound speed, m.s ${ }^{-1}$; Ag: Gas cross-sectional area, $\mathrm{m}^{2}$; As: Solid crosssectional area, $\mathrm{m}^{2}$; BR: Blockage ratio; CFD: Computational fluid dynamic; cp: Isobaric heat capacity, J.kg ${ }^{-1} . \mathrm{K}^{-1}$; cs: Solid heat capacity, J.kg ${ }^{-1} \cdot \mathrm{K}^{-1}$; DR: Drive ratio; fk: Rott's functions for temperature; fv: Rott's functions for viscosity; FFT: Fast Fourier transform; K: Thermal conductivity, W. $\mathrm{m}^{-1} . \mathrm{K}^{-1}$; L: Length, m; Lsn: Normalized stack length; I: Width, m; l0: Half plate thickness, m; p: Pressure amplitude, Pa; Pr: Prandtl number; Pac: Acoustic pressure, Pa; PSE: Plate surface engine; PSR: Plate surface refrigerator; QC: Cooling power, W; rh: Hydraulic radius, m; s: Entropy, $\mathrm{kg} \cdot \mathrm{m}^{2} \cdot \mathrm{s}^{-2} \cdot \mathrm{K}^{-1}$. T: Temperature, $\mathrm{K}$; TAE: Thermoacoustic engine; TAR: Thermoacoustic refrigerator; $\Delta T$ : Temperature difference, $K ; \nabla T$ : Temperature gradient, $K \cdot \mathrm{m}^{-1}$ : $\mathrm{u}$ : Velocity amplitude, $\mathrm{m} \cdot \mathrm{s}^{-1} ; \mathrm{W}$ : Acoustic power, W; xc: Stack center position, $m ; y 0$ : Half plate gap, $m$

\section{Authors' contributions}

Zahra Bouramdane: Conceptualization, Methodology, Software, Data curation, Formal analysis, Writing - original draft, Writing - review \& editing. Abdellah Bah: Work Supervision, Project administration, Conceptualization, Formal analysis, Results interpretation and analysis. Mohammed Alaoui: Work supervision, Project administration, Methodology. Nadia Martaj: Work supervision, Project administration, Methodology. The authors read and approved the final manuscript.

\section{Declarations}

\section{Competing interests}

The authors declare that they have no competing interests.

\section{Author details}

${ }^{1}$ Ecole Nationale Superieure des Arts et Metiers Rabat (ENSAM), Mohammed V University of Rabat, Avenue de I'Armee Royale, Madinat Al Irfane 10100, B.P. 6207 Rabat-Instituts, Rabat, Morocco. ${ }^{2}$ Graduate School of Engineering, EPF Troyes, 2 Rue F. Sastre, 10430 Rosieres-Pres-, Troyes, France.

\section{Published online: 28 February 2022}

\section{References}

1. Hofler, T. J. (1986). Thermoacoustic refrigerator design and performance. In Ph. D. thesis, Physics Department, University of Califomia at San Diego.

2. Atchley, A. A., Bass, H. E., Hofler, T. J., \& Lin, H. T. (1992). Study of a thermoacoustic prime mover below onset of self-oscillation. J Acoust Soc Am., 91(2), 734-743. https://doi.org/10.1121/1.402535

3. Swift, G. W. (1992). Analysis and performance of a large thermoacoustic engine. J Acoust Soc Am., 92(3), 1551-1563. https://doi.org/10.1121/1.403896

4. Arnott, W. P., Belcher, J. R., Raspet, R., \& Bass, H. E. (1994). Stability analysis of a helium-filled thermoacoustic engine. Acoust Soc Am J., 96(1), 370-375. https://doi.org/10.1121/1.410486

5. Swift, G. W., \& Keolian, R. M. (1993). Thermoacoustics in pin-array stacks. J Acoust Soc Am., 94(2), 941-943. https://doi.org/10.1121/1.408196

6. Hayden, M. E., \& Swift, G. W. (1997). Thermoacoustic relaxation in a pin-array stack. J Acoust Soc Am, 102(5). https://doi.org/10.1121/1.420325

7. Bösel, J., Trepp, C., \& Fourie, J. G. (1999). An alternative stack arrangement for thermoacoustic heat pumps and refrigerators. https:/doi.org/10.1121/1.427088

8. Adeff, J. A., Hofler, T. J., Atchley, A. A., \& Moss, W. C. (1998). Measurements with reticulated vitreous carbon stacks in thermoacoustic prime movers and refrigerators. J Acoust Soc Am., 104(1), 32-38. https://doi.org/10.1121/1.424 055
9. Adeff, J. A., \& Hofler, T. J. (2000). Design and construction of a solarpowered, thermoacoustically driven, thermoacoustic refrigerator. J Acoust Soc Am., 107(6), L37-L42. https://doi.org/10.1121/1.429324

10. Zoontjens, L., Howard, C. Q., Zander, A. C., \& Cazzolato, B. S. (2008). Numerical comparison of thermoacoustic couples with modified stack plate edges. Int J Heat Mass Transf., 51(19), 4829-4840. https://doi.org/10.1016/j. ijheatmasstransfer.2008.02.037

11. Matveev, K. I. (2010). Thermoacoustic energy analysis of transverse-pin and tortuous stacks at large acoustic displacements. Int J Therm Sci., 49(6), 10191025. https://doi.org/10.1016/j.jithermalsci.2009.12.007

12. Asgharian, B., \& Matveev, K. I. (2014). Influence of finite heat capacity of solid pins and their spacing on thermoacoustic performance of transverse-pin stacks. Appl Therm Eng., 62(2), 593-598. https://doi.org/10.1016/j.appltherma leng.2013.10.014

13. Zolpakar, N. A., Mohd-Ghazali, N., \& Ahmad, R. (2014). Simultaneous optimization of four parameters in the stack unit of a thermoacoustic refrigerator. Int J AirCond Refrig, 22(02), 1450011. https://doi.org/10.1142/S2010132514500114

14. Zolpakar, N. A., \& Mohd-Ghazali, N. (2019). Comparison of a thermoacoustic refrigerator stack performance: Mylar spiral, celcor substrates and 3D printed stacks. Int J Air-Cond Refrig, 27(03), 1950021. https://doi.org/10.1142/S201 0132519500214

15. Dragonetti, R., Napolitano, M., Di Filippo, S., \& Romano, R. (2016). Modeling energy conversion in a tortuous stack for thermoacostic applications. Appl Therm Eng., 103, 233-242. https://doi.org/10.1016/j.applthermaleng.2016.04.076

16. Abd El-Rahman, A. I., Abdelfattah, W. A., \& Fouad, M. A. (2017). A 3D investigation of thermoacoustic fields in a square stack. Int I Heat Mass Transf., 108, 292-300. https://doi.org/10.1016/j.j.jheatmasstransfer.2016.12.015

17. Yahya, S. G., Mao, X., \& Jaworski, A. J. (2017). Experimental investigation of thermal performance of random stack materials for use in standing wave thermoacoustic refrigerators. Int J Refrig., 75, 52-63. https://doi.org/10.1016/j. ijrefrig.2017.01.013

18. Napolitano, M., Romano, R., \& Dragonetti, R. (2017). Open-cell foams for thermoacoustic applications. Energy, 138, 147-156. https://doi.org/10.1016/j. energy.2017.07.042

19. Liu, L., Yang, P., \& Liu, Y. (2019). Comprehensive performance improvement of standing wave thermoacoustic engine with converging stack: Thermodynamic analysis and optimization. Appl Therm Eng., 160, 114096. https://doi.org/10.1016/j.applthermaleng.2019.114096

20. Chaiwongsa, P., \& Wongwises, S. (2021). Effect of the blockage ratios of circular stack on the performance of the air-based standing wave thermoacoustic refrigerator using heat pipe. Case Stud Therm Eng., 24, 100843. https://doi.org/10.1016/j.csite.2021.100843

21. Zolpakar, N. A., Mohd-Ghazali, N., \& Hassan El-Fawal, M. (2016). Performance analysis of the standing wave thermoacoustic refrigerator: a review. Renew Sustain Energy Rev., 54, 626-634. https://doi.org/10.1016/j.rser.2015.10.018

22. Swift, G. W. (2017). Thermoacoustics: a unifying perspective for some engines and refrigerators, 2nd ed. Springer International Publishing. https://doi.org/1 0.1007/978-3-319-66933-5

23. Tijani, M. E. H., Zeegers, J. C. H., \& de Waele, A. T. A. M. (2002). The optimal stack spacing for thermoacoustic refrigeration. J Acoust Soc Am., 112(1), 128-133. https://doi.org/10.1121/1.1487842

24. Bouramdane, Z., Zaaoumi, A., Bah, A., Martaj, N., \& ALAOUI, M. (2018). Numerical analysis of acoustic field in standing wave thermoacoustic engine with different stack lengths, 1-6. https://doi.org/10.1109/ECAl.2018.8679030

25. Bouramdane, Z., Bah, A., Alaoui, M., \& Martaj, N. (2021). CFD modeling and performance analysis of a thermoacoustically driven thermoacoustic refrigerator. Int J Air-Cond Refrig, 2150029. https://doi.org/10.1142/\$2010132 521500292

26. Swift, G. W. (1988). Thermoacoustic engines. J Acoust Soc Am., 84(4), 11451180. https://doi.org/10.1121/1.396617

27. MEH (Hassan), T. (2001). Loudspeaker-driven thermo-acoustic refrigeration. https://doi.org/10.6100/R547542

28. "COMSOL Multiphysics Reference Manual," p. 1742. https://www.comsol.fr/

29. Garrett, S. L. (1999). Reinventing the engine. Nature, 399(6734), 303-305. https://doi.org/10.1038/20546

30. Zink, F., Vipperman, J., \& Schäfer, L. (2008). Advancing thermoacoustics through CFD simulation using fluent, 8. https://doi.org/10.1115/IMECE2008-66510

\section{Publisher's Note}

Springer Nature remains neutral with regard to jurisdictional claims in published maps and institutional affiliations. 